\title{
Physical conditions in the central molecular zone inferred by $\mathrm{H}_{3}^{+}$
}

\author{
Franck Le Petit ${ }^{1}$, Maxime Ruaud ${ }^{2,3}$, Emeric Bron ${ }^{1}$, Benjamin Godard ${ }^{1}$, Evelyne Roueff ${ }^{1}$, \\ David Languignon ${ }^{1}$, and Jacques Le Bourlot ${ }^{1,4}$
}

\author{
${ }^{1}$ LERMA, Observatoire de Paris, PSL Research University, CNRS, Sorbonne Université, UPMC Paris 06, 92190 Meudon, France \\ e-mail: Franck. LePetit@obspm.fr \\ 2 Université Bordeaux, LAB, UMR 5804, 33270 Floirac, France \\ 3 CNRS, LAB, UMR 5804, 33270 Floirac, France \\ ${ }^{4}$ Université Paris-Diderot Paris 07, 75013 Paris, France
}

Received 2 June 2015 / Accepted 18 September 2015

\begin{abstract}
Context. The $\mathrm{H}_{3}^{+}$molecule has been detected in many lines of sight within the central molecular zone (CMZ) with exceptionally large column densities and unusual excitation properties compared to diffuse local clouds. The detection of the $(3,3)$ metastable level has been suggested to be the signature of warm and diffuse gas in the CMZ.

Aims. We aim to determine the physical conditions and processes in the $\mathrm{CMZ}$ that explain the ubiquitous properties of $\mathrm{H}_{3}^{+}$in this medium and to constrain the value of the cosmic-ray ionization rate.

Methods. We use the Meudon photodissociation region (PDR) code in which $\mathrm{H}_{3}^{+}$excitation has been implemented. We re-examine the relationship between the column density of $\mathrm{H}_{3}^{+}$and the cosmic-ray ionization rate, $\zeta$, up to large values of $\zeta$ in the frame of this full chemical model. We study the impact of the various mechanisms that can excite $\mathrm{H}_{3}^{+}$in its metastable state. We produce grids of PDR models exploring different parameters $(\zeta$, size of clouds, metallicity) and infer the physical conditions that best match the observations toward ten lines of sight in the CMZ. For one of them, Herschel observations of $\mathrm{HF}, \mathrm{OH}^{+}, \mathrm{H}_{2} \mathrm{O}^{+}$, and $\mathrm{H}_{3} \mathrm{O}^{+}$can be used as additional constraints. We check that the results found for $\mathrm{H}_{3}^{+}$also account for the observations of these molecules.

Results. We find that the linear relationship between $N\left(\mathrm{H}_{3}^{+}\right)$and $\zeta$ only holds up to a certain value of the cosmic-ray ionization rate, which depends on the proton density. A value $\zeta \sim 1-11 \times 10^{-14} \mathrm{~s}^{-1}$ explains both the large observed $\mathrm{H}_{3}^{+}$column density and its excitation in the metastable level $(3,3)$. This $\zeta$ value agrees with that derived from synchrotron emission and $\mathrm{Fe} \mathrm{K} \alpha$ line. It also reproduces $N\left(\mathrm{OH}^{+}\right), N\left(\mathrm{H}_{2} \mathrm{O}^{+}\right)$and $N\left(\mathrm{H}_{3} \mathrm{O}^{+}\right)$detected toward $\mathrm{Sgr} \mathrm{B} 2(\mathrm{~N})$. We confirm that the $\mathrm{CMZ}$ probed by $\mathrm{H}_{3}^{+}$is diffuse, $n_{\mathrm{H}} \lesssim$ $100 \mathrm{~cm}^{-3}$ and warm, $T \sim 212-505 \mathrm{~K}$. This warm medium is due to cosmic-ray heating. We also find that the diffuse component probed by $\mathrm{H}_{3}^{+}$must fill a large fraction of the $\mathrm{CMZ}$. Finally, we suggest the warm gas in the $\mathrm{CMZ}$ enables efficient $\mathrm{H}_{2}$ formation via chemisorption sites as in PDRs. This contributes to enhance the abundance of $\mathrm{H}_{3}^{+}$in this high cosmic-ray flux environment.
\end{abstract}

Key words. astrochemistry - ISM: molecules - cosmic rays - ISM: clouds - Galaxy: nucleus

\section{Introduction}

$\mathrm{H}_{3}^{+}$has been observed in a variety of environments: dense clouds (Geballe \& Oka 1996; McCall et al. 1999; Brittain et al. 2004; Gibb et al. 2010), diffuse clouds (McCall et al. 1998; Geballe et al. 1999; McCall et al. 2002, 2003; Indriolo et al. 2007; Indriolo \& McCall 2012) and even the nucleus of an extragalactic source (Geballe et al. 2006). A comprehensive review has been recently published (Oka 2013). The cosmic-ray ionization rate can be inferred using $\mathrm{H}_{3}^{+}(\mathrm{McC}$ all et al. 2002, 2003; Le Petit et al. 2004). Indriolo et al. (2007) and Indriolo \& McCall (2012) present an exhaustive study of $\mathrm{H}_{3}^{+}$observations and conclude that, in local diffuse clouds, the cosmic-ray ionization rate ${ }^{1}$ $\zeta$ presents variations with a mean value equals to $3.5 \times 10^{-16} \mathrm{~s}^{-1}$. The first detection of $\mathrm{H}_{3}^{+}$in the central molecular zone (CMZ; Geballe et al. 1999) showed a surprisingly large column density. Subsequent detections in the CMZ (Goto et al. 2002, 2008, 2011, 2013, 2014; Oka et al. 2005; Geballe \& Oka 2010) revealed that, in a radius of at least $100 \mathrm{pc}$ around $\mathrm{Sgr} \mathrm{A}^{*}, \mathrm{H}_{3}^{+}$presents not

\footnotetext{
1 We note $\zeta$ the cosmic-ray ionization rate of $\mathrm{H}_{2}$, expressed in $\mathrm{s}^{-1}$, and $\zeta_{1}$ the one of $\mathrm{H}$ with $\zeta_{1} \sim 0.5 \times \zeta$.
}

only large column densities but also a peculiar excitation since it is observable in its $(3,3)$ metastable level lying at $361 \mathrm{~K}$ above the ground rotational state (see Fig. 8). Oka \& Epp (2004) established that $N(3,3) / N(1,1)$ and $N(3,3) / N(2,2)$ ratios can be used to infer gas density and temperature. They demonstrated that a large fraction of the CMZ must be neutral diffuse warm gas with $n_{\mathrm{H}}<100 \mathrm{~cm}^{-3}$ and $T \sim 200-300 \mathrm{~K}$ (Oka \& Epp 2004; Oka et al. 2005; Goto et al. 2008). Two important questions arise from these unusual observations: 1) what is the cosmic-ray ionization rate required to explain these large amounts of $\mathrm{H}_{3}^{+}$; and 2) what is the heating source responsible for the warm medium. To answer the first question, Oka et al. (2005) and Goto et al. (2008) used a simple analytic relation between $N\left(\mathrm{H}_{3}^{+}\right)$and $\zeta$. Oka et al. (2005) concluded that $\zeta \sim(2-7) \times 10^{-15} \mathrm{~s}^{-1}$, i.e., a cosmic-ray ionization rate typically ten times higher than in local diffuse clouds. More recently, Yusef-Zadeh et al. (2013) used synchrotron emission and Fe I K $\alpha$ line to constrain $\zeta$. They found $\zeta_{1} \sim 5 \times 10^{-15} \mathrm{~s}^{-1}$ with synchrotron emission and $10^{-14} \mathrm{~s}^{-1}$ with the Fe I K $\alpha$ line. Finally, Herschel observations of $\mathrm{OH}^{+}, \mathrm{H}_{2} \mathrm{O}^{+}$, and $\mathrm{H}_{3} \mathrm{O}^{+}$in the $\mathrm{CMZ}$ suggest $\zeta_{1} \sim 10^{-14} \mathrm{~s}^{-1}$ toward Sgr B2(M) and $(\mathrm{N})$ (Indriolo et al. 2015). 
In this paper, we use the Meudon PDR (photodissociation region) $\operatorname{code}^{2}$ (Le Petit et al. 2006) to study the properties of $\mathrm{H}_{3}^{+}$in a medium submitted to a high cosmic-ray ionizing flux and we propose a scenario that explains both the large $\mathrm{H}_{3}^{+}$column densities observed in the CMZ and its excitation in the $(3,3)$ metastable level. In Sect. 2, we examine the relationship between $N\left(\mathrm{H}_{3}^{+}\right)$and the cosmic-ray ionization rate for a large range of $\zeta$ (from $10^{-17}$ to $10^{-12} \mathrm{~s}^{-1}$ ) and various densities. We also study the impact of several processes on $\mathrm{H}_{3}^{+}$abundance, such as the recombination of electrons on grains and $\mathrm{H}_{2}$ formation mechanisms. In Sect. 3, we present an analysis of $\mathrm{H}_{3}^{+}$excitation based on new $\mathrm{H}_{3}^{+}$excitation collisional rates with $\mathrm{H}_{2}$ computed by Gómez-Carrasco et al. (2012). We compare these new results to the pioneering study of Oka \& Epp (2004). We also study the possibility of $\mathrm{H}_{3}^{+}$excitation by nonthermal processes, such as IR pumping and excitation at formation. In Sect. 4 we present a scenario accounting for both $N\left(\mathrm{H}_{3}^{+}\right)$and $\mathrm{H}_{3}^{+}$excitation, as observed in the CMZ. We check that our results are consistent with other observational tracers of cosmic rays: $\mathrm{OH}^{+}, \mathrm{H}_{2} \mathrm{O}^{+}$ and $\mathrm{H}_{3} \mathrm{O}^{+}$absorptions in the $\mathrm{CMZ}$, synchrotron, and $\mathrm{Fe} \mathrm{K} \alpha$ line emission. Finally, we compare the various heating and cooling mechanisms in the CMZ according to our model. Our conclusions are summarized in Sect. 5.

\section{Abundance of $\mathrm{H}_{3}^{+}$in diffuse gas}

Cosmic-ray ionization is the starting point of chemistries involving $\mathrm{H}, \mathrm{D}, \mathrm{O}$, and $\mathrm{N}$ in diffuse and translucent clouds since these atoms cannot be photoionized by the interstellar radiation field (ISRF); see the review by Grenier et al. (2015). OH and HD have been recognized early (Hartquist et al. 1978; Federman et al. 1996) as potential indicators of the cosmic-ray ionization rate $\zeta_{1}$ and a value of $\sim 10^{-17} \mathrm{~s}^{-1}$ was derived from the analysis of the column densities of $\mathrm{OH}$ and HD found in local diffuse clouds. Recently, Bialy \& Sternberg (2015) studied the chemistry of $\mathrm{OH}$ for various $\zeta / n_{\mathrm{H}}$ and low metallicities. Detections of $\mathrm{H}_{3}^{+}$toward various diffuse lines of sight (McCall et al. 2002, 2003; Indriolo et al. 2007; Indriolo \& McCall 2012) lead to a significantly larger cosmic-ray ionization rate. This conclusion relies on a simple analytic relation between $N\left(\mathrm{H}_{3}^{+}\right)$and $\zeta$ derived from the chemical balance between formation and destruction of $\mathrm{H}_{3}^{+}$(see below). More recently, Indriolo et al. (2015) used Herschel observations of $\mathrm{OH}^{+}, \mathrm{H}_{2} \mathrm{O}^{+}$, and $\mathrm{H}_{3} \mathrm{O}^{+}$in a sample of Galactic diffuse clouds and inferred a mean value of the cosmic-ray ionization rate in the local medium of $\zeta_{1} \sim 1.78 \times 10^{-16} \mathrm{~s}^{-1}$, using the same simple analytic expression based on chemical networks.

In this section, we revisit the relationship between $N\left(\mathrm{H}_{3}^{+}\right)$ and $\zeta$ and extend it to large values of the cosmic-ray ionization rate, as those may exist in the Galactic center. We also highlight the indirect effect of two processes on $N\left(\mathrm{H}_{3}^{+}\right)$: the $\mathrm{H}_{2}$ formation rate and the recombination of electrons on grains.

\section{1. $N\left(H_{3}^{+}\right)$vs. $\zeta$ - the simple analytic expression}

The chemical network ${ }^{3}$ of $\mathrm{H}_{3}^{+}$is simple. Formation of $\mathrm{H}_{3}^{+}$involves the ionization of $\mathrm{H}_{2}$ by cosmic rays, followed by the

\footnotetext{
2 The Meudon PDR code is available at http://ism.obspm. fr

3 All reaction rates used in this paper come from KIDA database http://kida.obs.u-bordeaux1. fr (Wakelam et al. 2012) and the 2012 edition of the UMIST database http://udfa. ajmarkwick . net
}

reaction of $\mathrm{H}_{2}^{+}$with $\mathrm{H}_{2}$ :

$$
\begin{aligned}
l l \mathrm{H}_{2}+\text { cosmic rays } \longrightarrow \mathrm{H}_{2}^{+}+\mathrm{e}^{-} \quad k_{\zeta}=0.96 \times \zeta \mathrm{s}^{-1} \\
\mathrm{H}_{2}^{+}+\mathrm{H}_{2} \longrightarrow \mathrm{H}_{3}^{+}+\mathrm{H} \quad k_{1}=2.0 \times 10^{-9} \mathrm{~cm}^{3} \mathrm{~s}^{-1} .
\end{aligned}
$$

In diffuse environments, the main $\mathrm{H}_{3}^{+}$destruction reaction is the recombination with electrons,

$\mathrm{H}_{3}^{+}+\mathrm{e}^{-} \longrightarrow$ products $k_{\mathrm{e}}=6.7 \times 10^{-8}(T / 300)^{-0.52} \mathrm{~cm}^{3} \mathrm{~s}^{-1}$.

In dense media, destruction of $\mathrm{H}_{3}^{+}$by $\mathrm{CO}$ has to be considered as well.

Assuming this simple chemical network, constant densities, homogeneous medium and integrating over the whole cloud of depth $L$, the steady state $\mathrm{H}_{3}^{+}$column density $N\left(\mathrm{H}_{3}^{+}\right)$is

$N\left(\mathrm{H}_{3}^{+}\right)=0.96 \frac{\zeta L}{k_{\mathrm{e}}} \frac{f}{2 x_{\mathrm{e}}}$

with $f$ the molecular fraction defined as $2 N\left(\mathrm{H}_{2}\right) / N_{\mathrm{H}}$, where $N_{\mathrm{H}}=N(\mathrm{H})+2 \times N\left(\mathrm{H}_{2}\right)$ and with $x_{\mathrm{e}}=n\left(\mathrm{e}^{-}\right) / n_{\mathrm{H}}$ the electronic fraction.

If the molecular fraction, electronic fraction, and gas temperature are known or can be guessed, one may deduce $\zeta \times L$ from the measure of $N\left(\mathrm{H}_{3}^{+}\right)$. In local diffuse and translucent clouds, when available, the molecular fraction is derived from observations in absorption of $\mathrm{H}$ and $\mathrm{H}_{2}$ in the far $\mathrm{UV}$, and the gas temperature is deduced from the ratio of the two first $\mathrm{H}_{2}$ rotational levels $J=0$ and $J=1$ (Rachford et al. 2002). The electronic fraction is usually assumed to be given by $n\left(\mathrm{C}^{+}\right) / n_{\mathrm{H}}$. This expression has been extensively used by McCall et al. (2002, 2003), Indriolo et al. (2007, 2010), Indriolo \& McCall (2012) to deduce $\zeta$ in local diffuse clouds. The conclusion of these studies is $\zeta \sim 1.7 \times 10^{-16}-1.1 \times 10^{-15} \mathrm{~s}^{-1}$ (Indriolo \& McCall 2012) in diffuse clouds, a significantly larger value than previously deduced from $\mathrm{OH}$ and $\mathrm{HD}$ observations. On the other hand, a detailed simulation with the Meudon PDR code of the diffuse line of sight toward $\zeta$ Persei, where $\mathrm{OH}, \mathrm{HD}$, and $\mathrm{H}_{3}^{+}$have been detected concomitantly, leads to a value of the cosmic-ray ionization rate of $2.5 \times 10^{-16} \mathrm{~s}^{-1}$ (Le Petit et al. 2004), whereas the simple analytic derivation restricted to the analysis of $\mathrm{H}_{3}^{+}$concluded to a value of $\sim 1.2 \times 10^{-15} \mathrm{~s}^{-1}$ (McCall et al. 2003). The value obtained by Le Petit et al. (2004) relies on the modeling of 18 species detected toward $\zeta$ Persei with the Meudon PDR code (Le Petit et al. 2006) and the Paris-Durham shock code (Flower \& Pineau des Forets 1998). One major constraint is $N(\mathrm{OH})$, which is also proportional to $\zeta$, so that the high value of $\zeta$ derived by McCall et al. (2003) leads to a column density of $\mathrm{OH}$ that is too large compared to observations (Felenbok \& Roueff 1996; Roueff 1996).

Equation (4) has also been used to infer $\zeta \times L$ toward various sources located in the CMZ (Oka et al. 2005; Goto et al. 2008, 2011). Very restricted molecular information is available for these lines of sight, however; the molecular fraction is unknown and the electronic fraction is still assumed to be $n\left(\mathrm{C}^{+}\right) / n_{\mathrm{H}}$. Assuming $f=1$, an order of magnitude of $\zeta \sim(2-7) \times 10^{-15} \mathrm{~s}^{-1}$ is estimated by Oka et al. (2005).

\section{2. $N\left(H_{3}^{+}\right)$vs. $\zeta$ relationship - numerical models}

In this section, we test Eq. (4) with the Meudon PDR code (Le Petit et al. 2006) for diffuse cloud conditions. We consider different diffuse clouds defined by their total visual extinctions, $A_{\mathrm{V}}^{\max }=1$, proton densities (three densities are considered, $n_{\mathrm{H}}=50,100$, and $1000 \mathrm{~cm}^{-3}$ ), and illuminated on both 
Table 1. Input parameters used in the Meudon PDR code common to all models discussed in Sect. 2.2.

\begin{tabular}{lll}
\hline \hline Parameter & Value & Unit/Ref. \\
\hline Geometry & plane-parallel & \\
Size & $A_{\mathrm{V}}^{\max }=1$ & \\
Equation of state & isochoric & \\
Density & $50,100,1000$ & $\mathrm{~cm}^{-3}$ \\
ISRF scaling factor, $G_{0}$ & $1(2$ sides $)$ & $(1)$ \\
Cosmic-ray ionization rate, $\zeta$ & variable & $\mathrm{s}^{-1}$ \\
Metallicity, $Z$ & 1 & \\
Dust extinction curve & mean Galactic & $(2)$ \\
Mass grain/mass gas & $0.01 \times \mathrm{Z}$ & \\
Mass PAH/mass grain & $4.6 \times 10^{-2}$ & $(3)$ \\
$N_{\mathrm{H}} / E(B-V)$ & $5.8 \times 10^{21} / Z$ & $\mathrm{~cm}$ \\
$\mathrm{R}_{\mathrm{V}}-2 \mathrm{mag}^{-1}$ \\
Grain size distribution & 3.1 & $(4)$ \\
Minimum grain radius & $\propto r^{-3.5}$ & $(5)$ \\
Maximum grain radius & $1 \times 10^{-7}$ & $\mathrm{~cm}$ \\
\hline
\end{tabular}

References. (1) Mathis et al. (1983); (2) Fitzpatrick \& Massa (1986); (3) Draine \& Li (2007); (4) Seaton (1979); (5) Mathis et al. (1977).

Table 2. Elemental abundances. $Z$ is the metallicity.

\begin{tabular}{lll}
\hline \hline Element & Elemental abundance & Reference \\
\hline $\mathrm{He}$ & 0.1 & \\
$\mathrm{C}$ & $1.32 \times 10^{-4} \times Z$ & 1 \\
$\mathrm{O}$ & $3.19 \times 10^{-4} \times Z$ & 2 \\
$\mathrm{~S}$ & $1.86 \times 10^{-5} \times Z$ & 1 \\
$\mathrm{~N}$ & $7.50 \times 10^{-5} \times Z$ & 3 \\
$\mathrm{~F}$ & $1.8 \times 10^{-8} \times Z$ & 4 \\
\hline
\end{tabular}

References. (1) Savage \& Sembach (1996); (2) Meyer et al. (1998); (3) Meyer et al. (1997); (4) Snow et al. (2007).

sides by the isotropic ISRF expressed in Mathis units (Mathis et al. 1983). The gas temperature is computed at each position in the cloud taking detailed cooling and heating mechanisms into account (photoelectric effect, cosmic-ray heating, exothermic reactions, ...), as described in Le Petit et al. (2006) and Gonzalez Garcia et al. (2008). Our chemical network includes 165 species linked by 2850 chemical reactions. The code computes stationary state chemical abundances at each position using photodestruction rates determined by the radiative transfer and gas temperature computed by the thermal balance. Then, column densities are computed. To test Eq. (4) on a wide range of cosmic-ray ionization rate values, we ran models with $\zeta$ from $10^{-17}$ to $10^{-12} \mathrm{~s}^{-1}$. The input parameters are summarized in Tables 1 and 2. We also introduce the metallicity $Z$ as a multiplicative factor of the elemental abundances of heavy elements $(\mathrm{C}, \mathrm{N}, \mathrm{O}, \mathrm{S}, \mathrm{F}, \ldots)$ and of grain abundances. In this section, $Z$ is fixed to 1 , whereas in Sect. 4, which is dedicated to CMZ conditions, $Z$ is set to 3 .

$N\left(\mathrm{H}_{3}^{+}\right)$as a function of $\zeta$ is presented in Fig. 1 where each point corresponds to a model. $\mathrm{H}_{3}^{+}$column density increases with $\zeta$ only up to a maximum value of the cosmic-ray ionization rate, $\zeta^{\max }$. For moderate $\zeta$, the relationship is linear, in agreement with Eq. (4). Using the computed $f, x_{\mathrm{e}}$, and $T$, we checked that Eq. (4) gives an extremely good agreement with the numerical model in the linear increasing part. However, when Eq. (4) is used to estimate $\zeta$ with observations, the molecular fraction, electronic fraction, and gas temperature may have to be guessed, contrary to our numerical check where they are consistently computed. The maximum of $N\left(\mathrm{H}_{3}^{+}\right)$, reached at $\zeta=\zeta^{\text {max }}$, depends on the

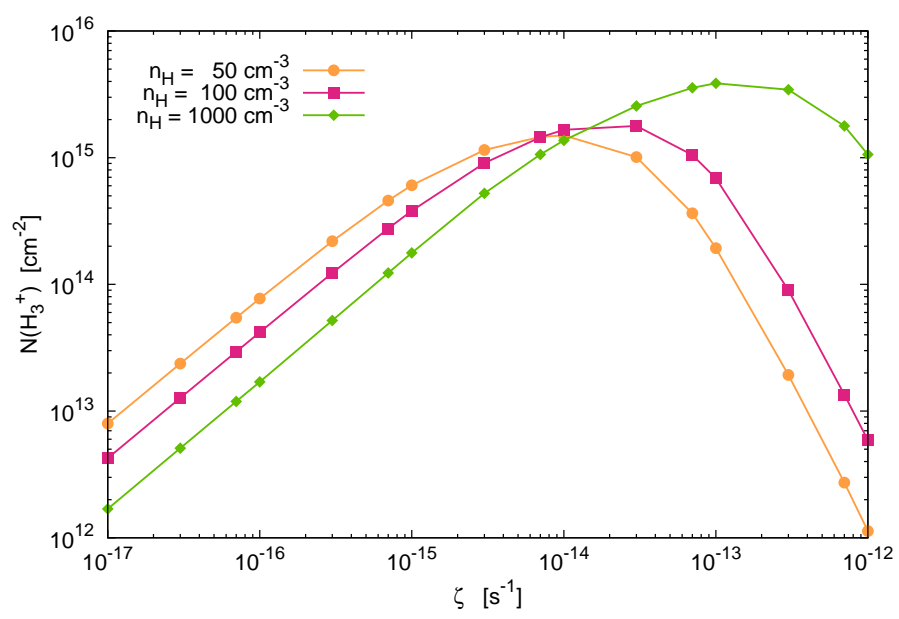

Fig. 1. Column density of $\mathrm{H}_{3}^{+}$as a function of $\zeta$. Each point corresponds to a PDR model as defined in Tables 1 and 2.

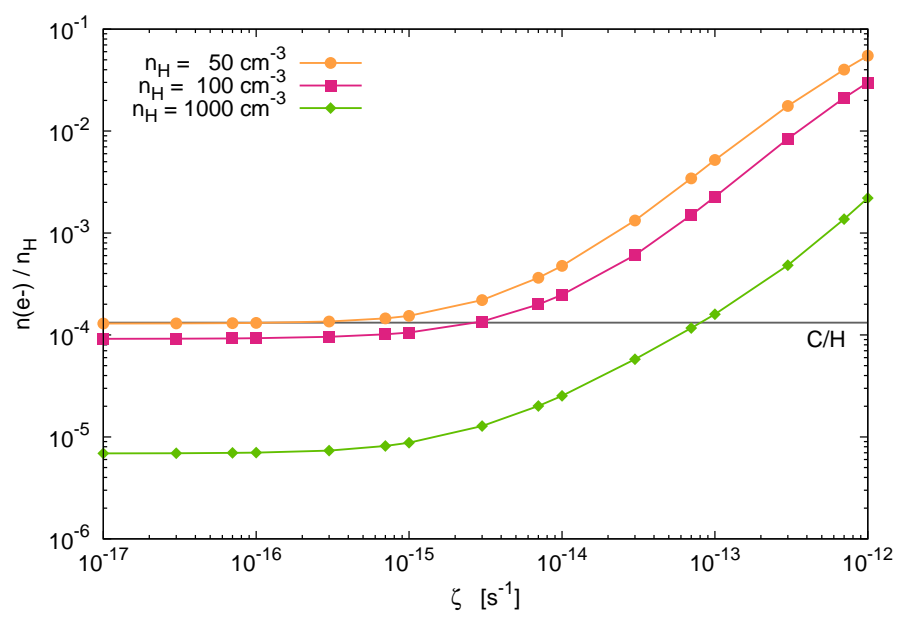

Fig. 2. Computed electronic fraction, $x_{\mathrm{e}}$, at $A_{\mathrm{V}}=0.5$ as a function of $\zeta$. Each point corresponds to a PDR model as defined in Tables 1 and 2 . The horizontal line represents the elemental abundance of carbon relative to $\mathrm{H}$.

gas density. In these models, the order of magnitude of the maximum of $N\left(\mathrm{H}_{3}^{+}\right)$is $10^{15} \mathrm{~cm}^{-2}$. This value depends on the size of the cloud, here $A_{\mathrm{V}}^{\max }=1$, and on the gas temperature (see below). For cosmic-rays ionization rates higher than (or even close to) $\zeta^{\max }$, Eq. (4) is obviously incorrect and cannot be used to deduce $\zeta$ from $N\left(\mathrm{H}_{3}^{+}\right)$. This decrease of $\mathrm{H}_{3}^{+}$column density with the cosmic-ray ionization rate can be understood from the behavior of the molecular and electronic fractions with $\zeta$.

Figure 2 shows the computed electronic fraction, $x_{\mathrm{e}}=$ $n\left(\mathrm{e}^{-}\right) / n_{\mathrm{H}}$, in the middle of the cloud $\left(A_{\mathrm{V}}=0.5\right)$ as a function of $\zeta$. The elemental abundance of $\mathrm{C}, \mathrm{C} / \mathrm{H}$, is a good proxy for $x_{\mathrm{e}}$ for low values of $\zeta$ and for low density clouds $\left(50\right.$ and $100 \mathrm{~cm}^{-3}$ in our examples). At high cosmic-ray fluxes, $x_{\mathrm{e}}$ is significantly higher than the $\mathrm{C} / \mathrm{H}$ ratio because a significant amount of electrons is produced by the cosmic-ray ionization of $\mathrm{H}$ and $\mathrm{H}_{2}$. At high densities and low cosmic-ray fluxes, $x_{\mathrm{e}}$ is lower than the $\mathrm{C} / \mathrm{H}$ ratio because of efficient neutralization of electrons on grains and polycyclic aromatic hydrocarbons (PAH). This point has been discussed by Liszt $(2003,2006)$.

Figure 3 illustrates the decrease of the molecular fraction with $\zeta$. The lower the density of the gas, the lower the $\zeta$ at which this decrease happens. At high flux of cosmic rays, molecular hydrogen is efficiently ionized by cosmic rays and forms $\mathrm{H}_{2}^{+}$, 


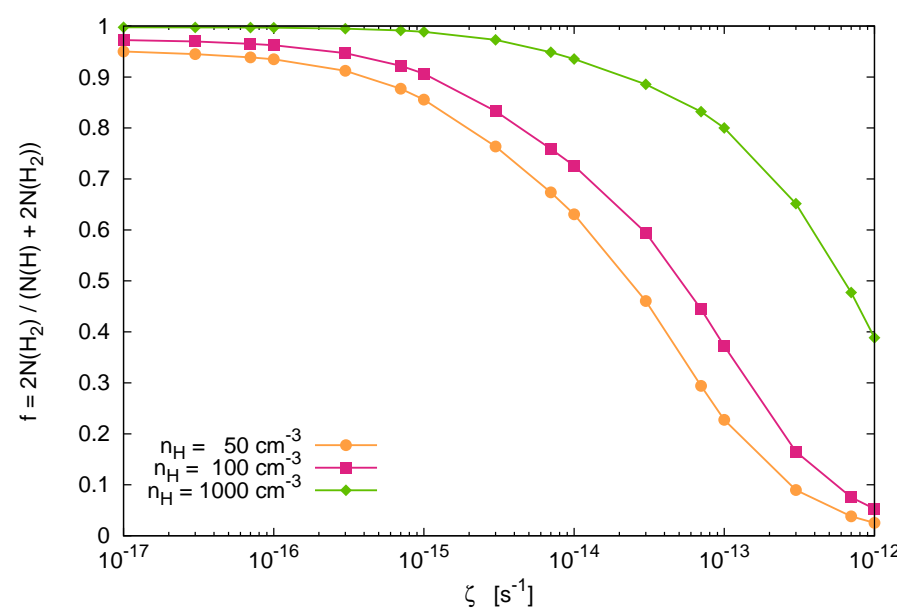

Fig. 3. Molecular fraction $f$ as a function of $\zeta$. Each point corresponds to a PDR model as defined in Tables 1 and 2 .

which quickly reacts with electrons to dissociate in hydrogen atoms or reacts with $\mathrm{H}$ and $\mathrm{H}_{2}$ to produce $\mathrm{H}_{2}$ and $\mathrm{H}_{3}^{+}$. Molecular fractions computed by the PDR code reach values up to $0.9-1$, whereas FUSE observations of local diffuse clouds show smaller molecular fractions, $f \sim 0.6$ (Rachford et al. 2002). The molecular fractions presented here correspond to single-cloud models with a total visual extinction of 1 . It is possible that several small components are present on FUSE lines of sight, resulting in a smaller total molecular fraction. This is consistent with a picture of fragmented diffuse interstellar gas (Godard et al. 2014).

The dependence of $N\left(\mathrm{H}_{3}^{+}\right)$with $\zeta$ is then straightforward to understand. When the cosmic-ray ionization rate is large, $N\left(\mathrm{H}_{3}^{+}\right)$ decreases with $\zeta$, first, because less $\mathrm{H}_{2}$ is available to form $\mathrm{H}_{3}^{+}$ and, second, because $\mathrm{H}_{3}^{+}$recombines efficiently because of a large abundance of electrons produced by cosmic-ray ionization of $\mathrm{H}$ and $\mathrm{H}_{2}$. A more general relationship between $N\left(\mathrm{H}_{3}^{+}\right)$and $\zeta$ than Eq. (4) can be obtained taking into account, in the chemical balance, $\mathrm{H}_{2}^{+}$reactions that are important at large $\zeta$ :

$$
\begin{aligned}
& \mathrm{H}_{2}^{+}+\mathrm{e}^{-} \longrightarrow \mathrm{H}+\mathrm{H} \quad k_{\mathrm{e}}\left(\mathrm{H}_{2}^{+}\right)=2.53 \times 10^{-7}(T / 300)^{-0.5} \mathrm{~cm}^{3} \mathrm{~s}^{-1} \\
& \mathrm{H}_{2}^{+}+\mathrm{H} \longrightarrow \mathrm{H}_{2}+\mathrm{H}^{+} \quad k_{2}=6.4 \times 10^{-10} \mathrm{~cm}^{3} \mathrm{~s}^{-1} .
\end{aligned}
$$

Then, the column density of $\mathrm{H}_{3}^{+}$in diffuse gas is written

$$
N\left(\mathrm{H}_{3}^{+}\right)=0.96 \times \frac{\zeta L}{k_{\mathrm{e}}} \frac{f}{2 x_{\mathrm{e}}}\left[1+\frac{2 k_{\mathrm{e}}\left(\mathrm{H}_{2}^{+}\right) x_{\mathrm{e}}}{k_{1} f}+\frac{2 k_{2}}{k_{1}}\left(\frac{1}{f}-1\right)\right]^{-1} .
$$

A similar equation is given by Indriolo \& McCall (2012) where destruction of $\mathrm{H}_{3}^{+}$by $\mathrm{CO}$ is included. Equation (5) shows that, as long as the expression in brackets is close to 1 (low $\zeta$ ), we recover the linear dependence between the column density of $\mathrm{H}_{3}^{+}$and $\zeta \times L$. However, when dissociative recombination of $\mathrm{H}_{2}^{+}$and charge transfer with $\mathrm{H}$ compete with destruction of $\mathrm{H}_{2}^{+}$by $\mathrm{H}_{2}$, Eq. (5) shows that the column density of $\mathrm{H}_{3}^{+}$is reduced compared to the prediction of Eq. (4).

Cosmic-ray ionization rates can thus only be deduced with the classical analytical expression, Eq. (4), for moderate $\zeta$, as in standard diffuse lines of sight. For large $\zeta$, this relationship fails. A more general expression, appropriate for low and high cosmicray fluxes in diffuse environments, is Eq. (5). In both cases, the difficulty to use such analytical expressions is to estimate properly $f$ and $x_{\mathrm{e}}$.

\subsection{Molecular fraction and $\mathrm{H}_{2}$ formation processes}

Because the molecular fraction is a key parameter in the relationship between the cosmic-ray ionization rate and $N\left(\mathrm{H}_{3}^{+}\right)$, the transition from atomic to molecular gas must be computed properly. The analytic theory of the $\mathrm{H}-\mathrm{H}_{2}$ transition has been described by Sternberg et al. (2014). In diffuse gas, $\mathrm{H}_{2}$ is destroyed by UV photons via absorption transitions in the Lyman and Werner bands followed by de-excitation in the continuum of the ground electronic state. In the models presented here, this process is computed taking continuum absorption by dust and carbon atoms of the UV radiation field and self-shielding of $\mathrm{H}_{2}$ lines into account (Le Petit et al. 2006). The formation of $\mathrm{H}_{2}$ takes place on grains. In numerical models, this can be simulated in very different ways, from simple analytic formulae to very detailed modeling. In most astrochemical models, a mean formation rate of $3 \times 10^{-17} \sqrt{T / 100} \mathrm{~cm}^{3} \mathrm{~s}^{-1}$ is used. This rate was deduced from Copernicus and FUSE observations of diffuse clouds in the local neighborhood (Jura 1974; Gry et al. 2002). Several studies based on ISO and Spitzer observations showed that this rate is too low to account for $\mathrm{H}_{2}$ emission lines in PDRs where the gas and the grains are warm (Habart et al. 2004, 2011). One may wonder if the mean formation rate determined in local diffuse lines of sight also applies to the $\mathrm{CMZ}$, where the gas is warm too. Indeed, this mean value hides complex microscopic mechanisms: $\mathrm{H}$ adsorption in chemisorption and physisorption sites, migration at the surface of grains and $\mathrm{H}_{2}$ formation. The mean value also hides the properties of grains and PAHs, such as their composition and temperature. In the Meudon PDR code, several formalisms are implemented to simulate $\mathrm{H}_{2}$ formation on grains: analytic expression (Le Petit et al. 2006), moment equation formalism (Le Petit et al. 2009), Langmuir-Hinshelwood (LH) and Eley-Rideal (ER) mechanisms (Le Bourlot et al. 2012), and a stochastic approach that considers the impact of grain temperature fluctuations on $\mathrm{H}_{2}$ formation rate (Bron et al. 2014).

In all models presented here, the $\mathrm{H}_{2}$ formation rate is computed using the formalism described in Le Bourlot et al. (2012), i.e., we take into account adsorption in physisorption and chemisorption sites with $\mathrm{H}_{2}$ formation via $\mathrm{LH}$ and ER mechanisms ${ }^{4}$. We upgraded the grain model assuming the grain size distribution contains a log-normal PAH component (Compiègne et al. 2011) plus a MRN power law (Mathis et al. 1977) for amorphous carbons and silicates with minimum and maximum radius equal to $10^{-7}$ and $3 \times 10^{-5} \mathrm{~cm}$. $\mathrm{H}_{2}$ formation rate on PAHs is not well known, but several laboratory experiments show that the process is efficient (Boschman et al. 2012; Mennella et al. 2012). Here, we assume that ER mechanism on PAHs is as efficient as on grains. As emphasized in Le Bourlot et al. (2012), the efficiency of the LH mechanism depends on grain temperatures whereas the efficiency of the ER mechanism depends on the gas temperature. Indeed, only $\mathrm{H}$ atoms with sufficient kinetic energy can reach chemisorption sites. In our models, we assume a threshold of $300 \mathrm{~K}$ to reach these sites. This low value accounts for the likely presence of defects on grain surfaces.

To study the sensitivity of the computed molecular fraction to the $\mathrm{H}_{2}$ formation model, we ran the same models as in the previous section with two prescriptions for the $\mathrm{H}_{2}$ formation rate on grains: the crude approximation, $3 \times 10^{-17} \sqrt{T / 100} \mathrm{~cm}^{3} \mathrm{~s}^{-1}$, and the more physical model taking ER and LH mechanisms

\footnotetext{
4 We have not used the most sophisticated $\mathrm{H}_{2}$ formation model at our disposal (Bron et al. 2014), which takes the effect of grain temperature fluctuations on $\mathrm{H}_{2}$ formation into account because it is too CPU time consuming. Nevertheless, we checked that grain temperature fluctuations do not have a strong effect on our conclusions.
} 


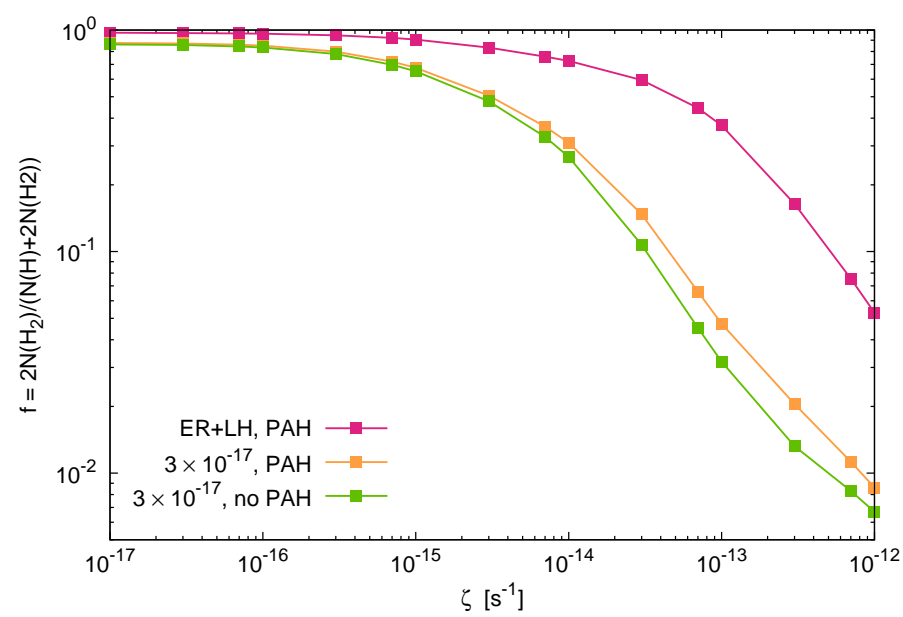

Fig. 4. Molecular fraction as a function of $\zeta$ for different grain distributions and $\mathrm{H}_{2}$ formation mechanisms. Each point corresponds to a PDR model as defined in Tables 1 and 2 with $n_{\mathrm{H}}=100 \mathrm{~cm}^{-3}$.

into account. Grain and PAH properties in the $\mathrm{CMZ}$ are poorly known. Since they affect the photoelectric heating and ionization degree through ions and electrons recombination and $\mathrm{H}_{2}$ formation, we also ran models with and without the PAH component. Computed $f$ as a function of $\zeta$ are presented for the $n_{\mathrm{H}}=100 \mathrm{~cm}^{-3}$ models in Fig. 4. In models with fixed $\mathrm{H}_{2}$ formation rate, the molecular fraction does not depend on the grain surface. There is only a slight $f$ increase at high $\zeta$ (between PAH and no PAH models) because of an increase of the gas temperature and variations of the available grain surface for charge recombinations. On the other hand, the consideration of ER mechanism leads to significantly higher molecular fractions at high $\zeta$ than the $3 \times 10^{-17} \sqrt{T / 100}$ approximation.

Figure 5 shows the gas temperature averaged over positions, $\langle T\rangle$, for all models presented in Sect. 2.2. For $\zeta<10^{-15} \mathrm{~s}^{-1}$ and diffuse conditions $\left(n_{\mathrm{H}} \leq 100 \mathrm{~cm}^{-3}\right),\langle T\rangle$ is $\sim 60 \mathrm{~K}$, in agreement with FUSE observations (Rachford et al. 2002). Increasing $\zeta$ from $10^{-17}$ to $10^{-12} \mathrm{~s}^{-1}$ increases the gas temperature from 60 to $1200 \mathrm{~K}$ in models with $n_{\mathrm{H}}=100 \mathrm{~cm}^{-3}$. In models with a large value of $\zeta$, cosmic rays heat the gas to several hundred Kelvin, firstly, by direct ionization of $\mathrm{H}$ and $\mathrm{H}_{2}$, followed by thermalization of electrons with the gas; and, secondly, because electrons recombine in exothermic dissociative recombination reactions (mostly the $\mathrm{H}_{3}^{+}+\mathrm{e}^{-}$reactions with $\Delta E=4.7 \mathrm{eV}$ in the three body dissociation). In our models, we assume that each cosmicray ionization contributes to the heating of the gas by $4 \mathrm{eV}$. Usually, formation of $\mathrm{H}_{2}$ via chemisorbed $\mathrm{H}$ atoms is efficient only in photodominated regions, where the temperature of the gas reaches several hundred Kelvin. In typical diffuse clouds, where the gas is at $\simeq 60 \mathrm{~K}$ (Rachford et al. 2002), this process is unlikely. The situation may be different for diffuse gas in the CMZ for which Oka \& Epp (2004) suggested that the temperature is several hundred Kelvin, enough to provide the kinetic energy for $\mathrm{H}$ atoms to reach chemisorption sites and increase the $\mathrm{H}_{2}$ formation rate by a significative amount compared to local diffuse clouds.

\subsection{Electronic fraction and $e^{-}$recombinations on grains}

The second important parameter in deriving $\zeta$ from $\mathrm{H}_{3}^{+}$observations is the electronic fraction (cf. Eqs. (4) and (5)). We showed that, at high cosmic-ray flux, electrons produced by ionization

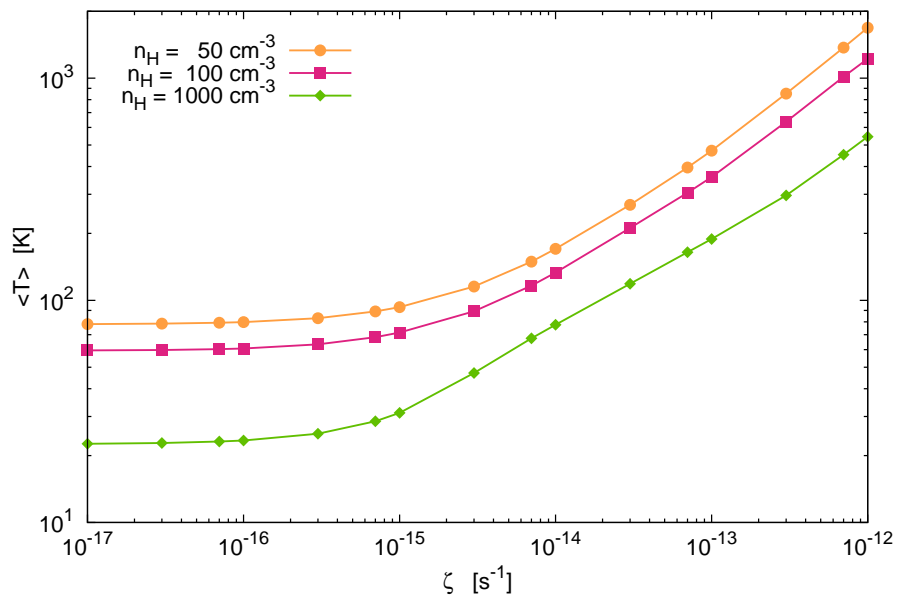

Fig. 5. Mean gas temperature for all models presented in Sect. 2.2 Each point corresponds to a PDR model as defined in Tables 1 and 2.

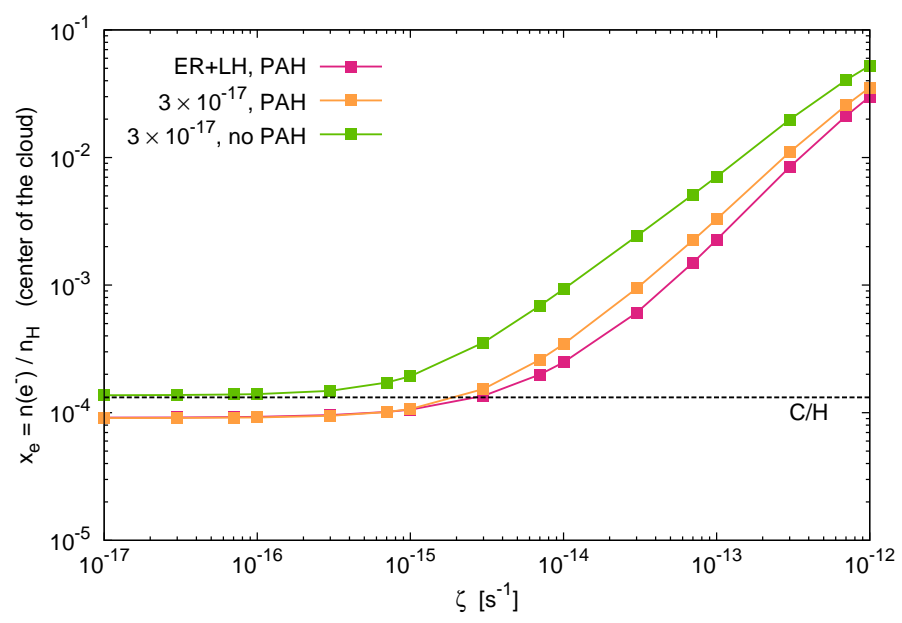

Fig. 6. Electronic fraction as a function of $\zeta$ for different grain distributions and $\mathrm{H}_{2}$ formation mechanisms. Each point corresponds to a PDR model as defined in Tables 1 and 2 with $n_{\mathrm{H}}=100 \mathrm{~cm}^{-3}$.

of $\mathrm{H}$ and $\mathrm{H}_{2}$ cannot be neglected compared to those produced by UV ionization of carbon and grains.

Figure 6 presents $x_{\mathrm{e}}$ as a function of $\zeta$ for the previous $n_{\mathrm{H}}=100 \mathrm{~cm}^{-3}$ models. The $\mathrm{H}_{2}$ formation formalism has only a small impact on $x_{\mathrm{e}}$. On the contrary, grain surface has a strong impact on $x_{\mathrm{e}}$. The higher the available grain surface to recombine electrons, the lower the electronic fraction. We do not know the grain properties in the CMZ. In our comparison of models to CMZ observations, we assume a standard grain size distribution plus a PAH component, as described in Sects. 2.2 and 2.3 and we scale the grain mass with the metallicity in the CMZ.

\subsection{Effect of the gas temperature}

Warm gas contributes to increase the abundance of $\mathrm{H}_{3}^{+}$(Le Petit et al. 2004). First, as described above, it increases $\mathrm{H}_{2}$ formation rate by ER mechanism on chemisorption sites. The available $\mathrm{H}_{2}$ may lead to the formation of $\mathrm{H}_{3}^{+}$. Second, the dissociative recombination rate of $\mathrm{H}_{3}^{+}$decreases with $T$. Third, at $T$ above $100 \mathrm{~K}$, the charge exchange reaction between $\mathrm{O}$ and $\mathrm{H}^{+}$becomes efficient, i.e.,

$\mathrm{H}^{+}+\mathrm{O} \longrightarrow \mathrm{O}^{+}+\mathrm{H} \quad k=7.31 \times 10^{-10}(T / 300)^{0.23} \mathrm{e}^{-226 / T}$, 


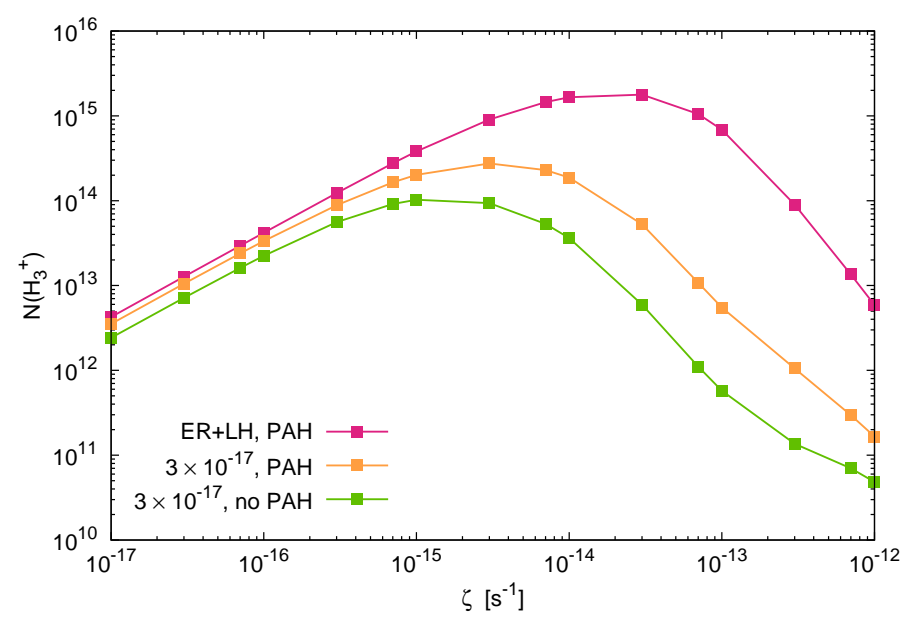

Fig. 7. $N\left(\mathrm{H}_{3}^{+}\right)$as a function of $\zeta$ for the two $\mathrm{H}_{2}$ formation models $\left(3 \times 10^{-17} \sqrt{T / 100} \mathrm{~cm}^{3} \mathrm{~s}^{-1}\right.$ and Langmuir-Hinshelwood plus EleyRideal mechanisms) and two grain distributions: MRN-like distribution and MRN-like + PAH distribution. For all models $n_{\mathrm{H}}=100 \mathrm{~cm}^{-3}$.

with $k$ in $\mathrm{cm}^{3} \mathrm{~s}^{-1}$. This opens the chemical network of oxygen hydride ions $\mathrm{OH}^{+}, \mathrm{H}_{2} \mathrm{O}^{+}$, and $\mathrm{H}_{3} \mathrm{O}^{+}$formed through successive reactions with $\mathrm{H}_{2}$. An increase of the densities of these hydrides provides additional channels for electrons consumption. Hence, at large temperatures, electrons have several ways to recombine efficiently in competition with $\mathrm{H}_{3}^{+}$recombination. Moreover, it is then not surprising to find similar velocity profiles in $\mathrm{H}_{3}^{+}$and $\mathrm{H}_{2} \mathrm{O}^{+}$spectra obtained in two nearby CMZ sources, the $\iota$ star in the Galactic center and Sgr B2 (Schilke et al. 2013), as emphasized by Oka (2015). We specifically discuss the link between $\mathrm{H}_{3}^{+}$and oxygen hydride ions in Sect. 4.2. As a result of these temperature dependence effects on $\mathrm{H}_{3}^{+}$, it is also less surprising to observe large $\mathrm{H}_{3}^{+}$column densities in the warm diffuse gas of the CMZ.

\subsection{Conclusion on $\mathrm{N}\left(\mathrm{H}_{3}^{+}\right)$and $\zeta$}

To summarize the effect of $\mathrm{H}_{2}$ formation prescription and grain/PAHs properties, Fig. 7 presents the dependence of $N\left(\mathrm{H}_{3}^{+}\right)$ on $\zeta$ for the $n_{\mathrm{H}}=100 \mathrm{~cm}^{-3}$ models. The position of the maximum of the $N\left(\mathrm{H}_{3}^{+}\right)-\zeta$ relationship depends strongly on the $\mathrm{H}_{2}$ formation prescription and on grain/PAHs characteristics. A too simplistic recipe, such as the $3 \times 10^{-17} \sqrt{T / 100}$ value, leads to erroneous conclusions when trying to deduce $\zeta$ from $\mathrm{H}_{3}^{+}$observations for high values of $\zeta$. This illustrates the crucial need, in interstellar chemical studies, to account for detailed microphysical processes.

\section{3. $\mathrm{H}_{3}^{+}$excitation}

Gas density and temperature in diffuse gas can be inferred from $\mathrm{H}_{3}^{+}$observations in states $(3,3),(2,2)$, and $(1,1)$ (Oka \& Epp 2004). Geballe (2012) stated that $N(3,3) / N(1,1)$ is a good thermometer for any temperature and $N(3,3) / N(2,2)$ is a good densimeter for high temperature; see discussion in Oka (2013). Oka \& Epp (2004) computed maps of these ratios in the plane $n\left(\mathrm{H}_{2}\right)-T$ and deduced that the gas probed by $\mathrm{H}_{3}^{+}$in the $\mathrm{CMZ}$ is diffuse $\left(n\left(\mathrm{H}_{2}\right) \leq 70 \mathrm{~cm}^{-3}\right)$ and warm $(T \geq 300 \mathrm{~K})$. Their computation takes radiative de-excitations and collisional excitation and de-excitation with $\mathrm{H}_{2}$ into account. As $\mathrm{H}_{3}^{+}$collision rates were not available, Oka \& Epp (2004) proposed an expression based on the Langevin expression with proper account of microreversibility. Recent theoretical studies have been performed on the $\mathrm{H}_{3}^{+}-\mathrm{H}_{2}$ system. First, Hugo et al. (2009) computed reactive and nonreactive collision rates for temperatures below $50 \mathrm{~K}$ with a strong ergodicity and full nuclear spin scrambling hypothesis. Second, Gómez-Carrasco et al. (2012) extended the temperature range up to $500 \mathrm{~K}$ and used a dynamically biased statistical model coupled to a recent global potential energy surface of the $\mathrm{H}_{5}^{+}$system (Aguado et al. 2010). Finally, Park \& Light (2007) studied the ortho-para conversion in the $\mathrm{H}_{3}^{+}+\mathrm{H}_{2}$ reaction.

Apart from thermal processes, two additional excitation mechanisms of the $(3,3)$ metastable level may have to be considered. As reported by Goto et al. (2008), John Black suggested the possibility of IR pumping of the ortho $(1,0)$ that could decay after several transitions in the $(3,3)$ metastable level. Another excitation mechanism could be the formation of $\mathrm{H}_{3}^{+}$in excited states as a result of the exothermic $\mathrm{H}_{2}^{+}+\mathrm{H}_{2}$ reaction.

In this section, we examine the relevance of various excitation mechanisms and discuss the relationship between $\mathrm{H}_{3}^{+}$excitation and the physical conditions of the gas, temperature, and density. We introduced the various $\mathrm{H}_{3}^{+}$excitation mechanisms in the Meudon PDR code. Radiative data come from Lindsay $\&$ McCall (2001) and are available electronically on the website $^{5}$ maintained by B. McCall. To discuss the uncertainties arising from the uncertainties in the collision rates, we implemented the two sets of collision rates by Oka \& Epp (2004) and Gómez-Carrasco et al. (2012). Whereas Oka \& Epp (2004) do not discriminate between ortho and para levels, Gómez-Carrasco et al. (2012) consider the appropriate nuclear spin restrictions and provide state-to-state collision rates for the 24 first levels of $\mathrm{H}_{3}^{+}$with $\mathrm{H}_{2}$. We implemented collisions of $\mathrm{H}_{3}^{+}$with $\mathrm{H}_{2}$ in $v=0, J=0$ and $v=0, J=1$ since most of molecular hydrogen molecules are in these low energy states. We always use 24 levels for $\mathrm{H}_{3}^{+}$except in Sect. 3.3 where 54 levels are introduced, as required for our analysis. Collision rates with $\mathrm{H}$ and $\mathrm{He}$ are derived from those with $\mathrm{H}_{2} J=0$ with a scaling law involving the reduced mass. Since $\mathrm{H}_{3}^{+}$is coexistent with $\mathrm{H}_{2}, \mathrm{H}_{2}$ is the main collisional excitation and de-excitation partner. Collisions with electrons have been studied (Faure \& Tennyson 2003; Faure et al. 2006; Kokoouline et al. 2010) and may contribute to $\mathrm{H}_{3}^{+}$excitation. Because of the electronic potential interaction symmetries, collisional excitation due to electrons may only occur between levels $(J, K)$ with the same values of $K$. As the $(3,3)$ metastable level is the lowest level with $K=3$, contribution of electron collisions may only take place via $(4,3)$ de-excitation involving an energy difference of $494 \mathrm{~K}$. In the present study, we did not include those collisions in the excitation balance.

We recall that the Meudon PDR code computes the atomic and molecular structure of a 1D plane-parallel slab of dust and gas. By default, gas and grain temperatures are computed at each position in the cloud taking the various local heating and cooling mechanisms into account in parallel to level excitation of several atoms and molecules responsible for the cooling of the gas. Level excitation is computed by solving the radiative transfer equation as described in Gonzalez Garcia et al. (2008). The PDR code considers collisional excitation and de-excitation, radiative de-excitation, radiative pumping, chemical formation, and destruction. It is also possible to run isothermal models with a fixed gas temperature. This alternative is used in Sect. 3.2 to produce maps in the plane $n_{\mathrm{H}}-T$, which can be compared to Oka \& Epp (2004).

http://h3plus.illinois.edu 


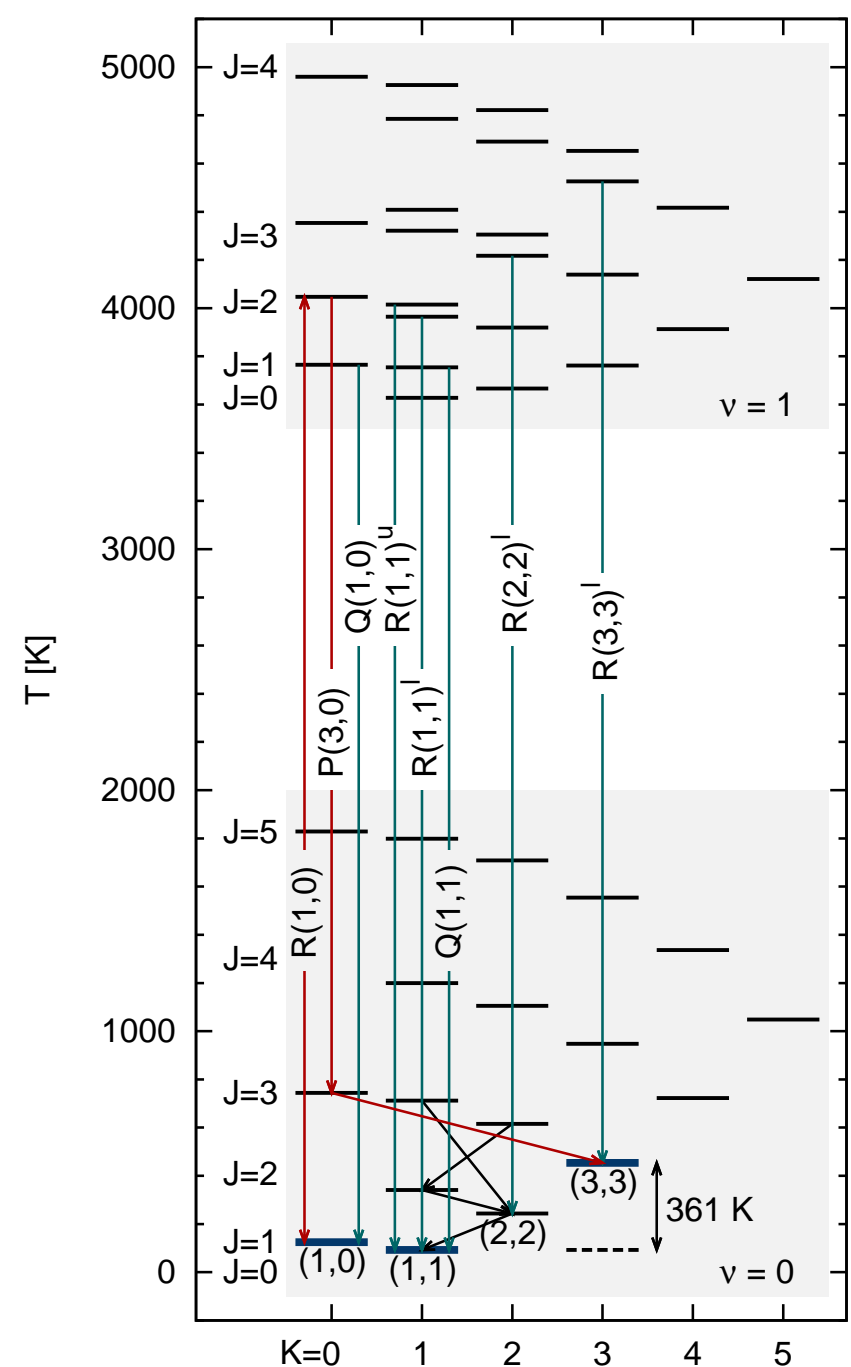

Fig. 8. Schema of $\mathrm{H}_{3}^{+}$levels with the IR pumping mechanism discussed in Sect. 3.3 in red.

\subsection{Chemical excitation}

The exothermicity of the $\mathrm{H}_{2}+\mathrm{H}_{2}^{+}$reaction is $1.7 \mathrm{eV}$. This energy may be redistributed as kinetic energy of products and internal energy of $\mathrm{H}_{3}^{+}$and can contribute to the excitation of its $(3,3)$ level. This mechanism may be efficient if the timescale associated with the formation-destruction cycle is short compared to other (de)excitation mechanisms. The chemical timescale of $\mathrm{H}_{3}^{+}$is inversely proportional to the product of the destructive recombination rate and $\mathrm{H}_{3}^{+}$density and thus dependent on the cosmic ionization rate. In the center of a stationary diffuse cloud $\left(n_{\mathrm{H}}=100 \mathrm{~cm}^{-3}\right.$, ISRF scaling factor $\left.G_{0}=1, A_{\mathrm{V}}^{\max }=1\right)$, we obtain a chemical timescale of about 19 yrs for $\zeta=10^{-16} \mathrm{~s}^{-1}$ and 2 yrs for $\zeta=10^{-13} \mathrm{~s}^{-1}$. For comparison, the collisional deexcitation rate of the $(3,3)$ level is found to be $\sim 3$ yrs and $\sim 4$ yrs, respectively. Then, chemical excitation may contribute and even dominate $\mathrm{H}_{3}^{+}$excitation at large $\zeta$.

Since $\mathrm{H}_{3}^{+}$excitation at formation is unknown, we consider three different prescriptions:

- Scenario A: we assume equipartition of energy, i.e., $33 \%$ of the available $1.7 \mathrm{eV}$ are included as internal energy (corresponding to $6510 \mathrm{~K}$ ). This energy is far above the 24 first energy levels for which excitation rates are available (Gómez-Carrasco et al. 2012). In addition, these

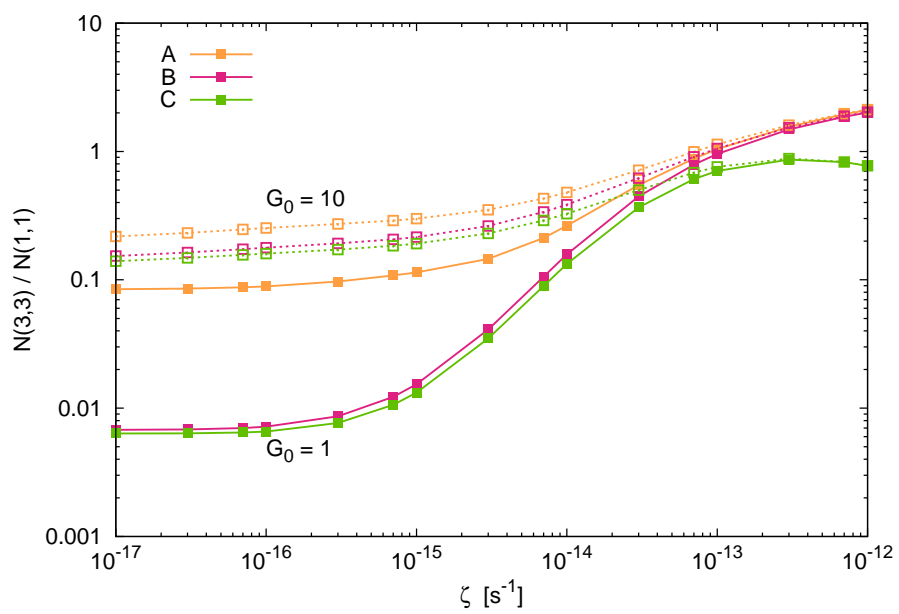

Fig. 9. Ratios $N(3,3) / N(1,1)$ as a function of $\zeta$ for three scenarii for $\mathrm{H}_{3}^{+}$excitation at formation. A: $33 \%$ of the exothermicity is used as internal energy, $\mathrm{B}: \mathrm{H}_{3}^{+}$is formed following a Boltzmann distribution at gas temperature, $\mathrm{C}: \mathrm{H}_{3}^{+}$is formed in its two levels $(1,1)$ and $(1,0)$ in a ratio corresponding to their degeneracy. Solid lines correspond to $G_{0}=1$ and dotted lines to $G_{0}=10$.

excited levels decay radiatively at a rate $\sim 10^{-3} \mathrm{~s}^{-1}$ (Lindsay $\&$ McCall 2001), much more efficiently than through any collisional deexcitation process. We then assume that these high levels radiatively cascade toward lower levels and finally contribute to the population of the 24 first levels proportionally to their statistical weights.

- Scenario $\mathrm{B}: \mathrm{H}_{3}^{+}$is formed following a Boltzmann distribution at gas temperature. This hypothesis assumes the newly formed molecule has time to thermalize with the gas.

- Scenario $C$ : formation energy is transferred mainly into kinetic energy and $\mathrm{H}_{3}^{+}$molecules are formed in their para $(1,1) /$ ortho $(1,0)$ ground states, in the ratio of their statistical weights.

Figure 9 shows the $N(3,3) / N(1,1)$ ratios as a function of $\zeta$ for the three prescriptions. Results are presented for PDR models with $n_{\mathrm{H}}=100 \mathrm{~cm}^{-3}, A_{\mathrm{V}}^{\max }=1$, and $G_{0}=1$ and 10 . This last value of $G_{0}$ corresponds to the order of magnitude of the UV radiation field in the CMZ (Porter \& Strong 2005; Moskalenko et al. 2006). In these models, Gómez-Carrasco et al. (2012) collision rates are used.

The $N(3,3) / N(1,1)$ ratio exhibits a significant increase for low values of $\zeta$ when scenario $\mathrm{A}$ is introduced and when $G_{0}=1$. These conditions correspond to a mean kinetic temperature of $\sim 65 \mathrm{~K}$ for $\zeta=10^{-16} \mathrm{~s}^{-1}$ so that collisions are not efficient to excite the $(3,3)$ level. However, when $G_{0}=10$ under the same low values of $\zeta$, the mean kinetic temperature increases $(T$ reaches $\sim 160 \mathrm{~K}$ for $\left.\zeta=10^{-16} \mathrm{~s}^{-1}\right)$, such that the $N(3,3) / N(1,1)$ ratio also increases significantly. Hence, the additional excitation introduced in scenario $\mathrm{A}$ is moderate compared to that in scenarios $\mathrm{B}$ and $\mathrm{C}$.

At high $\zeta, N(3,3) / N(1,1)$ ratios increase with $\zeta$. In scenario $A$, the ratio converges toward $14 / 3$, the ratio of the statistical weights of the $(3,3)$ and $(1,1)$ levels since characteristic timescales for statistical equilibrium are controlled by chemical processes. Models $\mathrm{B}$, with the hypothesis that $\mathrm{H}_{3}^{+}$is formed following a Boltzmann distribution at gas temperature, also converge toward this value. Indeed, the mean gas temperature is then $360 \mathrm{~K}$ and $420 \mathrm{~K}$ for, respectively, $G_{0}=1$ and 10 , and for $\zeta=$ $10^{-13} \mathrm{~s}^{-1}$, so the Boltzmann distribution at gas temperature tends toward a repartition following statistical weights. Since the gas 

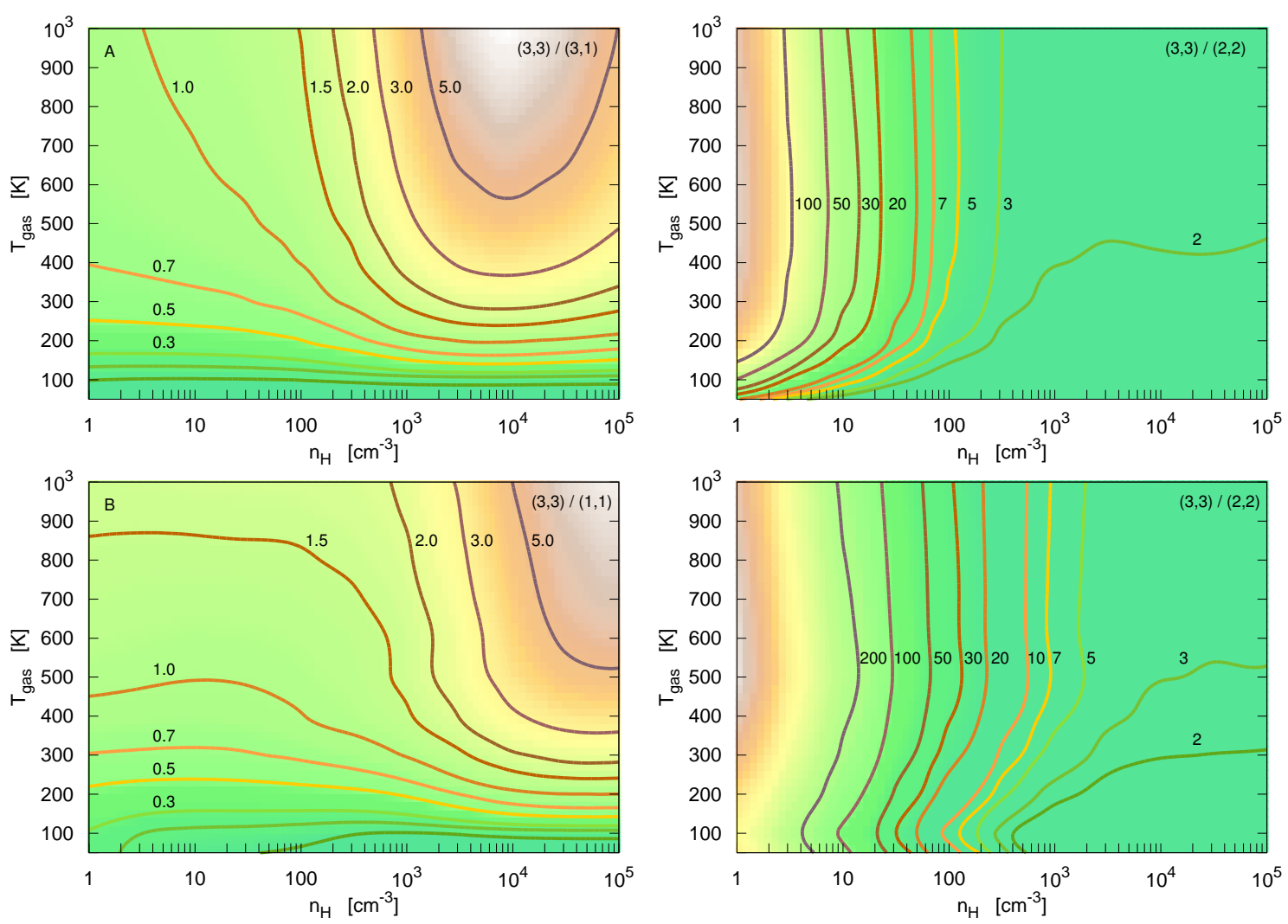

Fig. 10. Ratios $N(3,3) / N(1,1)$ and $N(3,3) / N(2,2)$ in the plane $n_{\mathrm{H}}-T$ obtained with the Meudon PDR code. Top (A): models with Oka \& Epp (2004) $\mathrm{H}_{3}^{+}$collision rates. Bottom (B): models with Gómez-Carrasco et al. (2012) collision rates.

temperature is high and collisional excitation is efficient, even scenario $\mathrm{C}$ gives large values of $N(3,3) / N(1,1)$. Nevertheless, for this scenario, when $\zeta>10^{-13} \mathrm{~s}^{-1}, N(3,3) / N(1,1)$ decreases with $\zeta$ because collisional excitations no longer have time to contribute to the excitation from low levels $(1,1)$ and $(1,0)$ before the molecule is destroyed.

These results show the importance of chemical excitation to compute $\mathrm{H}_{3}^{+}$excitation. Fortuitously, this contribution is not as decisive in $\mathrm{CMZ}$ conditions $\left(G_{0} \simeq 10\right.$ and high flux of cosmic rays) as in typical diffuse clouds because gas temperature becomes high enough to enhance collisional excitations. Values close to 1 have been found for the $N(3,3) / N(1,1)$ ratio in CMZ observations. These values are obtained in Fig. 9 for high values of $\zeta$. This is discussed in Sect. 4. In the following, scenario A is always used except when explicitly said.

\subsection{Sensitivity to collisional excitation rates}

Collisional excitation of $\mathrm{H}_{3}^{+}$is a significant source of uncertainty in describing the population of excited levels. We check the sensitivity to the available collision rates by running two sets of 176 isothermal and isochoric PDR models, one set with Oka \& Epp (2004) prescription and another one with Gómez-Carrasco et al. (2012) collision rates. The proton density is varied from 1 to $10^{5} \mathrm{~cm}^{-3}$ and the temperature range is between 50 and $1000 \mathrm{~K}$. The Galactic values reported in Tables 1 and 2 are used $\left(G_{0}=1\right.$, $\left.A_{\mathrm{v}}^{\max }=1\right)$ with a cosmic-ray ionization rate value $\zeta=10^{-16} \mathrm{~s}^{-1}$. We also assume that $33 \%$ of the exothermicity of the formation reaction excites $\mathrm{H}_{3}^{+}$at formation following scenario A described in Sect. 3.1.

Figure 10 shows corresponding results as contour maps of the ratios $N(3,3) / N(1,1)$ and $N(3,3) / N(2,2)$. First, we obtain

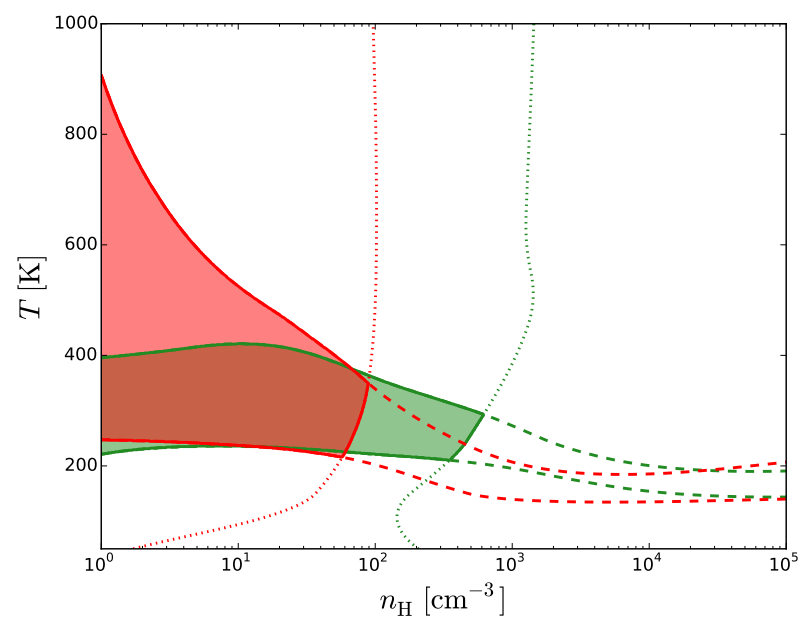

Fig. 11. $N(3,3) / N(1,1)$ (dashed lines) and $N(3,3) / N(2,2)$ (dotted lines) ratios in a plane $n_{\mathrm{H}}-T$ matching observations toward 2MASS J17470898-2829561, $\Delta v$ between -150 and $-60 \mathrm{~km} \mathrm{~s}^{-1}$ (Goto et al. 2011). Red corresponds to models obtained with Oka \& Epp (2004) collision rates and green to models obtained with Gómez-Carrasco et al. (2012) collision rates.

similar results to those reported in Oka \& Epp (2004) and Oka et al. (2005) when we use their collision rates prescription, even though our models include chemical excitation. Second, we also find that models including Gómez-Carrasco et al. (2012) collision rates suggest slightly higher densities and temperatures than those obtained with Oka \& Epp (2004) prescription. This is more apparent in Fig. 11, which presents the space parameter where $N(3,3) / N(1,1)=0.57 \pm 0.11$ and $N(3,3) / N(2,2)>3.21$, 

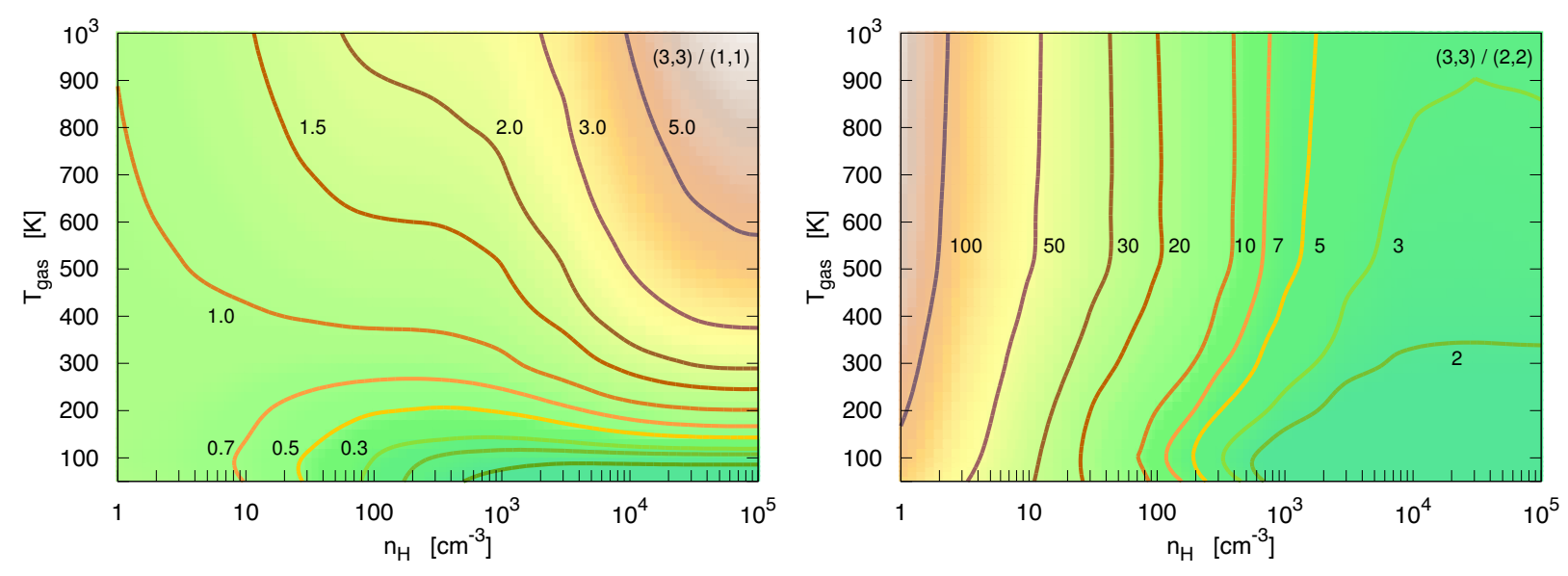

Fig. 12. Same as bottom panels of Fig. 10, but produced with models with $\zeta=3 \times 10^{-14} \mathrm{~s}^{-1}$.

corresponding to the 2MASS J17470898-2829561 line of sight (Goto et al. 2011). We then conclude that, to the first order, the available collisional excitation rates of $\mathrm{H}_{3}^{+}$(Oka \& Epp 2004; Gómez-Carrasco et al. 2012) lead to similar estimates of the density and temperature. In the following, we use Gómez-Carrasco et al. (2012) collision rates, which are calculated from a precise intermolecular potential surface and take nuclear spin restriction rules into account.

Figure 12 presents the same ratios for models with a larger value of $\zeta, 3 \times 10^{-14} \mathrm{~s}^{-1}$, which is more appropriate for CMZ conditions, as emphasized later. Compared to the previous contour maps, the increase of the cosmic-ray flux enhances the $N(3,3) / N(1,1)$ ratio in the low density $\left(n_{\mathrm{H}}<100 \mathrm{~cm}^{-3}\right)$ and low temperature $(T<300 \mathrm{~K})$ domain because of the faster formation-destruction cycle of $\mathrm{H}_{3}^{+}$.

\subsection{Excitation by IR pumping}

Goto et al. (2008) reported a suggestion by John Black: $(3,3)$ level population may be enhanced by IR pumping (see Fig. 8). Photons at $3.6685 \mu \mathrm{m}$ pump the ortho $(1,0)$ level of the ground vibrational state toward the vibrationally $v_{2}=1 \mathrm{ex}-$ cited state $(2,0)$. This is followed by rapid radiative de-excitation into $(3,0)$ in $2.53 \times 10^{-2} \mathrm{~s}$. Then level $(3,0)$ de-excites toward the $(3,3)$ metastable level through a semiforbidden radiative transition in less than four hours. Goto et al. (2008) estimate that, despite an enhanced IR radiation field in the CMZ compared to local conditions, this process should not be competitive with collisional excitation in the CMZ (see their Sect. 5.5).

To examine the impact of this process, we increase the number of $\mathrm{H}_{3}^{+}$levels considered in the PDR code up to level $v=1$, $J=2, K=0$. We use Gómez-Carrasco et al. (2012) collision rates for the 24 first levels of $\mathrm{H}_{3}^{+}$and Oka \& Epp prescription for upper levels. Following Porter \& Strong (2005) and Moskalenko et al. (2006), the radiation field in the $\mathrm{CMZ}$ at $3.7 \mu \mathrm{m}$ could be 20 times higher than in local ISM. In the Meudon PDR code, the intensity of the incident radiation field in the visible and infrared is modeled by two black bodies: one corresponding to stars emission and one to dust emission. We scale the intensities of these black bodies so that the intensity in the visible - IR impinging on the slab of gas is 20 times the standard radiation field at the wavelength of the $R(1,0)$ transition.

We ran several models of diffuse clouds with $n_{\mathrm{H}}=100 \mathrm{~cm}^{-3}$, $A_{\mathrm{V}}^{\max }=1$ and with different values of $G_{0}, \zeta$ and incident intensity at $3.6685 \mu \mathrm{m}$ to cover local and CMZ conditions. Table 3
Table 3. Effect of IR pumping on $N(3,3) / N(1,1)$ and on $N(3,3) / N(2,2)$.

\begin{tabular}{lllll}
\hline \hline$G_{0}$ & $\zeta$ & $\mathrm{IR}$ & $N(3,3) / N(1,1)$ & $N(3,3) / N(2,2)$ \\
\hline 1 & $10^{-16}$ & $\times 1$ & 0.1 & 7.5 \\
1 & $10^{-16}$ & $\times 20$ & 0.1 & 5.9 \\
1 & $10^{-13}$ & $\times 1$ & 1.0 & 9.4 \\
1 & $10^{-13}$ & $\times 20$ & 0.9 & 7.7 \\
10 & $10^{-16}$ & $\times 1$ & 0.4 & 8.6 \\
10 & $10^{-16}$ & $\times 20$ & 0.4 & 8.1 \\
10 & $10^{-13}$ & $\times 1$ & 1.2 & 8.6 \\
10 & $10^{-13}$ & $\times 20$ & 1.2 & 8.2 \\
\hline
\end{tabular}

Notes. The first three columns correspond to input parameters. IR is the scaling factor to the IR part of the ISRF.

presents $N(3,3) / N(1,1)$ and $N(3,3) / N(2,2)$ for these models. We find that IR pumping always has negligible impact on the $N(3,3) / N(1,1)$ ratio. It has a minor effect on the ratio $N(3,3) / N(2,2)$ in standard conditions. In CMZ conditions, $G_{0}=10$ and $\zeta=10^{-13} \mathrm{~s}^{-1}$, IR pumping has no effect compared to the other excitation processes (direct collisions and excitation at formation). These models confirm the statement of Goto et al. (2008). In the following of the paper, we neglect this process and use models with $24 \mathrm{H}_{3}^{+}$levels, allowing us to use consistent collision rates by Gómez-Carrasco et al. (2012).

\section{Physical conditions in the CMZ}

In this section, we try to constrain the physical conditions and processes in the CMZ, including the cosmic-ray ionization rate. We ran a grid of 1776 isochoric PDR models with parameters relevant to the $\mathrm{CMZ}$ conditions. The metallicity, $Z$, in the $\mathrm{CMZ}$ is between 2 and 5 times higher than in the solar neighborhood (Rolleston et al. 2000; Daflon \& Cunha 2004; Rudolph et al. 2006). We set it to $Z=3$. This increases elemental and grain abundances as described in Tables 1 and 2. In all models, the incident radiation field in the far-UV impinging on both sides of the cloud is the ISRF scaled by a factor ${ }^{6} G_{0}=10$ (Porter \& Strong 2005). The grid of models is divided in five sets of proton densities, $n_{\mathrm{H}}=10,20,50,100,1000 \mathrm{~cm}^{-3}$. For each proton density, $\zeta$ ranges from $10^{-17}$ to $10^{-12} \mathrm{~s}^{-1}$, and the size of the clouds,

\footnotetext{
6 In Sect. 3.3, we tested with the maximal possible value, $G_{0}=20$ to study the possible impact of IR pumping. Here, we use an intermediate value, $G_{0}=10$. This has no impact on the results.
} 

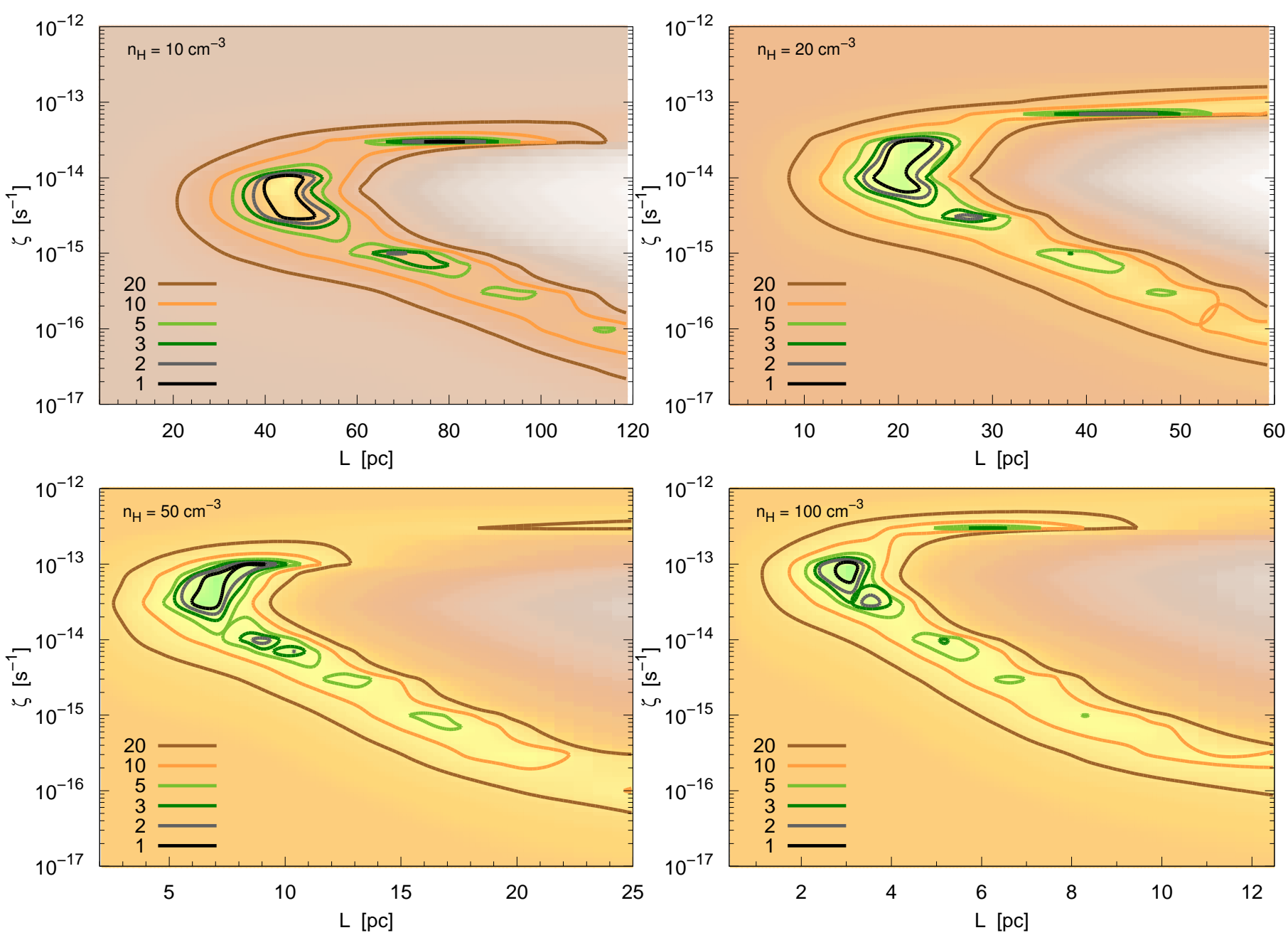

Fig. 13. Contour map of $\chi^{2}$ using $\mathrm{H}_{3}^{+}$column densities $N(1,1)$ and $N(3,3)$ toward $\mathrm{J} 1747$ for the four densities.

$A_{\mathrm{V}}^{\max }$, ranges from 0.2 to 6 . We take the full physics described above into account except for the enhanced IR pumping of $\mathrm{H}_{3}^{+}$, since we found that this process has a negligible effect on $\mathrm{H}_{3}^{+}$excitation. This simplification allows us to limit the computation of $\mathrm{H}_{3}^{+}$excitation to its 24 first levels for which collision rates are reliable. In all models, the gas temperature is computed at each position of the cloud taking heating and cooling mechanisms implemented in the Meudon PDR code into account. For models with constant density, the relationship between the size in $\mathrm{cm}, L$, and the visual extinction, $A_{\mathrm{V}}^{\max }$, is $L=6.1 A_{\mathrm{V}}^{\max } / Z\left(100 / n_{\mathrm{H}}\right) \mathrm{pc}$.

\subsection{Estimates of $\zeta$ and $L$ with $H_{3}^{+}$}

To infer physical conditions on each line of sight where $\mathrm{H}_{3}^{+}$is detected, we use a $\chi^{2}$ minimization built with $\mathrm{H}_{3}^{+}$column densities in levels $(1,1),(3,3)$, and $(1,0)$ when known. We also check that the best models according to the minimization procedure are compatible with the upper limits on the $(2,2)$ level. Table 4 summarizes $\mathrm{H}_{3}^{+}$observed column densities on the $10 \mathrm{CMZ}$ lines of sight on which $\chi^{2}$ minimizations are performed.

For all lines of sight, except GCS 3-2, a clear $\chi^{2}<1$ zone is found with models $n_{\mathrm{H}}=10$ to $100 \mathrm{~cm}^{-3}$ (Fig. 13 shows an illustration of the $\chi^{2}$ minimization for the J1747 line of sight). Models with $n_{\mathrm{H}}=1000 \mathrm{~cm}^{-3}$ only reach a $\chi^{2}<1$ for GCS IRS 21, NHS 22, and, marginally, in the case of J1747. Moreover, when a $\chi^{2}$ of 1 is reached for $n_{\mathrm{H}}=1000 \mathrm{~cm}^{-3}$ models,
Table 4. Observed column densities of $\mathrm{H}_{3}^{+}$in the CMZ in $(1,1),(1,0)$, $(3,3)$, and $(2,2)$ levels.

\begin{tabular}{lcccc}
\hline \hline Source & $N(1,1)$ & $N(1,0)$ & $N(3,3)$ & $N(2,2)$ \\
\hline GC IRS 21 & $28.1 \pm 12.3$ & $9.0 \pm 4.4$ & $24.2 \pm 12.1$ & $<8.3$ \\
GC IRS 3 & $10.8 \pm 2.1$ & $3.4 \pm 1.3$ & $8.4 \pm 1.9$ & $<1.6$ \\
GC IRS 1W & $18.1 \pm 3.8$ & $7.9 \pm 2.4$ & $11.7 \pm 3.0$ & $<3.3$ \\
GCS 3-2 & $17.0 \pm 1.7$ & $4.6 \pm 0.8$ & $9.8 \pm 1.6$ & $<3.0$ \\
J1743 & $12.8 \pm 1.9$ & - & $5.7 \pm 2.2$ & $<2.1$ \\
J1747 & $6.0 \pm 0.7$ & - & $4.3 \pm 1.3$ & $<0.8$ \\
NHS 21 & $8.9 \pm 2.2$ & $3.7 \pm 1.5$ & $5.6 \pm 1.4$ & $<2.3$ \\
NHS 22 & $16.9 \pm 5.6$ & $7.9 \pm 3.5$ & $9.7 \pm 2.7$ & $<3.6$ \\
NHS 25 & $11.4 \pm 5.9$ & $4.0 \pm 3.8$ & $7.4 \pm 5.3$ & $<3.9$ \\
NHS 42 & $17.7 \pm 5.1$ & $8.4 \pm 4.1$ & $8.6 \pm 4.1$ & $<4.0$ \\
Mean & $14.5 \pm 0.8$ & $6.5 \pm 1.1$ & $8.6 \pm 1.0$ & \\
\hline
\end{tabular}

Notes. Column densities are expressed in $10^{14} \mathrm{~cm}^{-2}$.

References. J1747 corresponds to 2MASS J17470898-2829561 and J1743 to 2MASS J17432173-2951430. Data are from Goto et al. (2008) for all lines of sight except for J1747 and J1743 for which data come from Goto et al. (2011). The GCS 3-2 line of sight is not taken into account to compute the weighted mean value (see text).

column densities in the $(2,2)$ level are only lower than the upper limit by a few percent. For these reasons, even if it is formally not possible to reject models with densities of $1000 \mathrm{~cm}^{-3}$, this density is less likely. As expected, we find that the lower the density, 


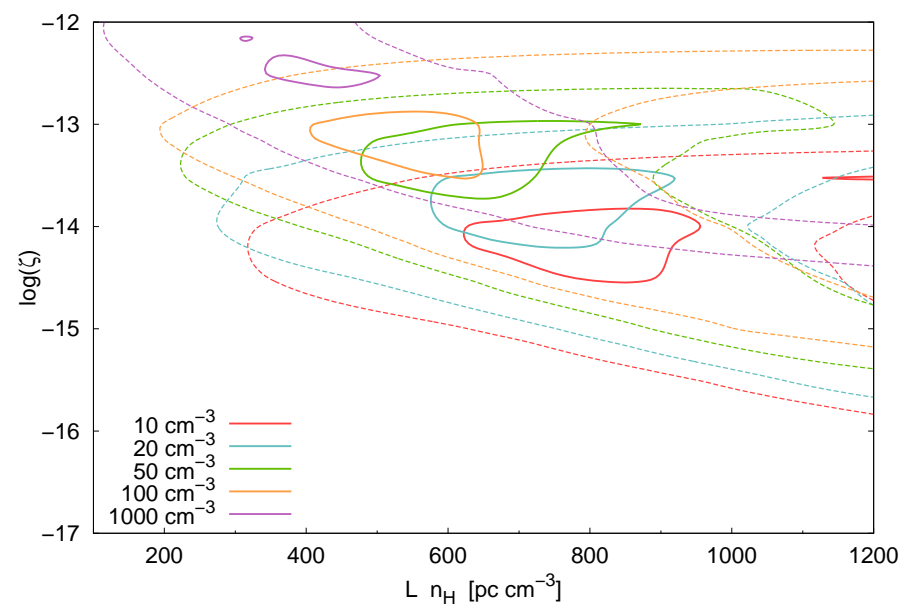

Fig. 14. Comparison of the $\chi^{2}$ contours for the five densities for the NHS 22 line of sight. Solid lines correspond to $\chi^{2}=1$ and dashed lines to $\chi^{2}=5$.

the lower the cosmic-ray ionization rate and the larger the size of the cloud. Figure 14 presents a comparison of $\chi^{2}$ contours for the five densities for the line-of-sight NHS 22.

Table 5 lists, for each line of sight, the positions in the $\zeta-L$ plane where $\chi^{2}$ values are minimal, as well as the corresponding computed $\mathrm{H}_{3}^{+}$level column densities, molecular fractions, average temperatures, and electronic fractions. Assuming $\mathrm{H}_{3}^{+}$has similar properties on the various lines of sight as demonstrated by Goto et al. $(2005,2008,2011)$, we can derive a weighted mean of observed column densities (Table 4). This weighted mean is computed assuming the uncertainties in observations are dominated by photon noise and therefore, follow a Poisson process. We exclude from this weighted mean the GCS 3-2 line of sight since it seems peculiar. A $\chi^{2}$ minimization on this weighted mean leads to a cosmic-ray ionization rate and a path length ranging from $10^{-14} \mathrm{~s}^{-1}$ and $66 \mathrm{pc}$ for $n_{\mathrm{H}}=10 \mathrm{~cm}^{-3}$, to $11 \times 10^{-14} \mathrm{~s}^{-1}$ and $4 \mathrm{pc}$ for $n_{\mathrm{H}}=100 \mathrm{~cm}^{-3}$ (see Table 5). It is difficult to constrain $\zeta$ and $L$ further without additional information about the gas density.

Our value of $\zeta$ is somewhat larger than the estimate by Yusef-Zadeh et al. (2013), who deduced from synchrotron and Fe K $\alpha$ observations, $\zeta_{1} \sim 10^{-15}-10^{-14} \mathrm{~s}^{-1}$. However, this value is not incompatible since low-energy cosmic-ray ions may contribute to ionization without producing synchrotron emission and, according to Strong et al. (2010), the luminosity of cosmicray protons is $\sim 70$ times that of cosmic-ray electrons.

\subsection{Additional constraints: $\mathrm{OH}^{+}, \mathrm{H}_{2} \mathrm{O}^{+}, \mathrm{H}_{3} \mathrm{O}^{+}$, and $\mathrm{HF}$}

Thanks to PRISMAS and HEXOS Herschel key programs, several hydride molecules $\left(\mathrm{HF}, \mathrm{CH}, \mathrm{OH}^{+}, \mathrm{H}_{2} \mathrm{O}^{+}, \mathrm{H}_{3} \mathrm{O}^{+}, \mathrm{CH}^{+}\right.$, $\left.\mathrm{SH}^{+}, \ldots\right)$ have been observed in absorption toward several lines of sight in the CMZ (Gerin et al. 2010a,b; Neufeld et al. 2010; Godard et al. 2012), but not on the lines of sight where $\mathrm{H}_{3}^{+}$is detected. Nevertheless, the Sgr B2(N) line of sight, part of the HEXOS program, is close to J1747. The projected distance between the two objects is $18 \mathrm{pc}$ (assuming a distance to the Galactic center of $8 \mathrm{kpc}$ ). Oka (2015) noticed a strong similarity between $\mathrm{H}_{2} \mathrm{O}^{+}$velocity components toward Sgr B2(M) and those of $\mathrm{H}_{3}^{+}$toward $\mathbf{J 1 7 4 7}$, and Indriolo et al. (2015) observe (see their Figs. 4 and 5) similar components between Sgr B2(N) and Sgr B2(M). Figure 15 presents HF and $\mathrm{H}_{3}^{+}$spectra toward Sgr B2 $(\mathrm{N})$ and J1747. Both molecules present similar

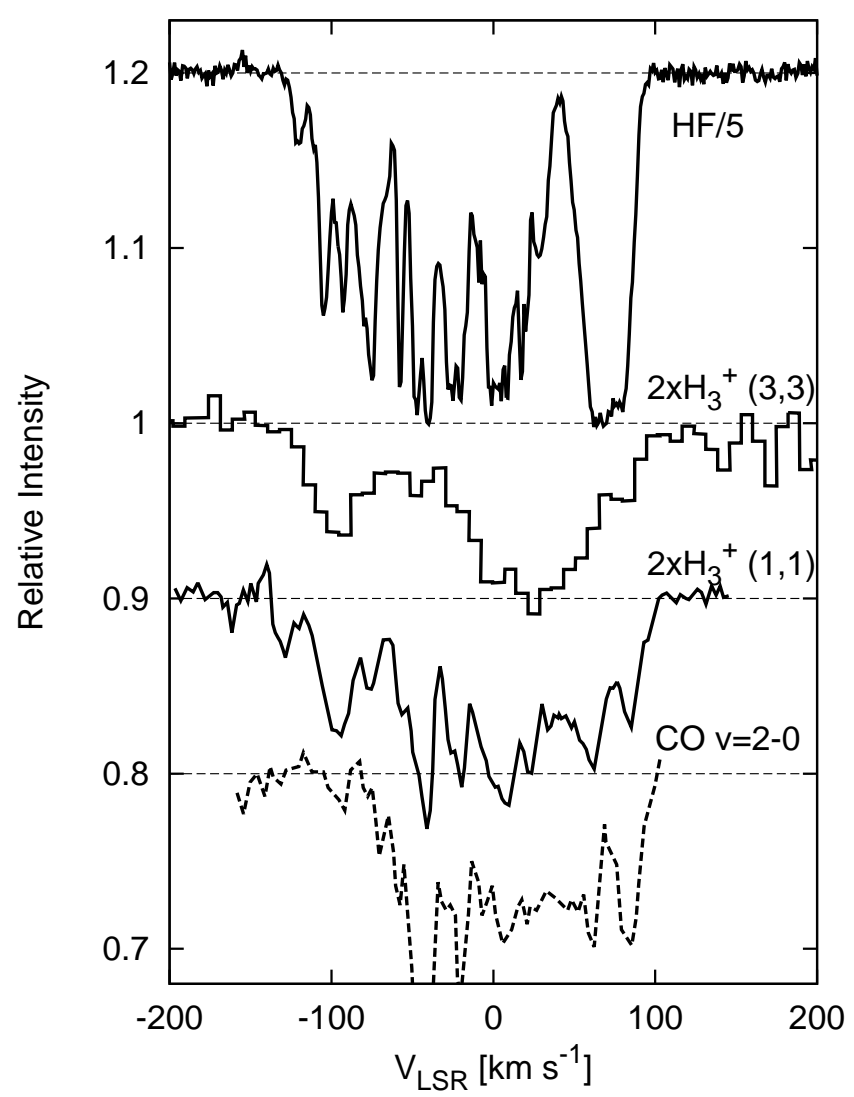

Fig. 15. HF spectrum for the $J=1-0$ transition obtained with Herschel/HIFI toward Sgr B2(N) (HEXOS key program) and $\mathrm{H}_{3}^{+}$spectrum for the $R(3,3)$ transition (Goto et al. 2011) and for the $R(1,1)^{l}$ (Geballe \& Oka 2010) toward $\mathrm{J} 1747$. The $\mathrm{H}_{3}^{+}$spectrum of the $R(3,3)$ transition has been corrected by the CO absorption. See Sect. 2 in Goto et al. (2011) for more details.

velocity absorption components. Absorption features associated with the diffuse gas in the CMZ are located between -150 and $-60 \mathrm{~km} \mathrm{~s}^{-1}$ (Goto et al. 2011). In this velocity range, HF presents four absorption components, $\mathrm{H}_{3}^{+}$one unresolved $(3,3)$ absorption feature and $3(1,1)$ components. In addition, weak $\mathrm{CO}$ absorption components are detected. Concerning the other absorption components, the strong absorption of $\mathrm{HF}$ at $v_{\mathrm{LSR}}=$ $60 \mathrm{~km} \mathrm{~s}^{-1}$ is related to the Sgr B2(N) complex. Three absorption components due to spiral arms are present at $-40,-20$, and $0 \mathrm{~km} \mathrm{~s}^{-1}$, deduced from a longitude-velocity map of the CO $J=1-0$ emission in this region (Oka et al. 2012).

These similarities in the velocity components between -150 and $-60 \mathrm{~km} \mathrm{~s}^{-1}$ may indicate that the size of the diffuse component containing $\mathrm{H}_{3}^{+}$, toward J1747, extends up to the Sgr B2(N) line of sight and, therefore, the size of this component should be at least of $\sim 18 \mathrm{pc}$. This is also compatible with the fact that $\mathrm{H}_{3}^{+}$is largely observed in the $\mathrm{CMZ}$ with always the same peculiar properties, indicating that the filling factor of the neutral diffuse gas probed by $\mathrm{H}_{3}^{+}$must be large. According to Table 5, this leads to $n_{\mathrm{H}}<50 \mathrm{~cm}^{-3}$ for the $\mathrm{J} 1747$ line of sight. As a consequence, the cosmic-ray ionization rate should be $\zeta<5 \times 10^{-14} \mathrm{~s}^{-1}$.

Assuming that observations toward J1747 and Sgr B2(N) probe the same diffuse gas, derived column densities toward Sgr B2(N) can be used to test our models. We reanalyzed Herschel HF observations. Column densities in the four components between -150 and $-60 \mathrm{~km} \mathrm{~s}^{-1}$ are given in Table 6 . As discussed by Godard et al. (2012), N(HF) may provide an upper 
Table 5. Results of the best models defined as those minimizing $\chi^{2}$ for the various densities, $n_{\mathrm{H}}=10,20,50$, and $100 \mathrm{~cm}^{-3}$.

\begin{tabular}{|c|c|c|c|c|c|c|c|c|c|c|}
\hline \multicolumn{11}{|c|}{$n_{\mathrm{H}}=10 \mathrm{~cm}^{-3}$} \\
\hline Source & $\zeta$ & $L$ & $\chi^{2}$ & $N(1,1)$ & $N(1,0)$ & $N(3,3)$ & $N(2,2)$ & $f$ & $\langle T\rangle$ & $\left\langle x_{\mathrm{e}}\right\rangle$ \\
\hline GC IRS 21 & 1 & 94 & 0.6 & 19.5 & 11.5 & 12.8 & 0.14 & 0.5 & 316 & 1.3 \\
\hline GC IRS 3 & 3 & 108 & 0.3 & 9.6 & 4.4 & 9.4 & 0.11 & 0.4 & 471 & 3.0 \\
\hline GC IRS 1W & 1 & 81 & 0.3 & 15.8 & 9.2 & 10.4 & 0.11 & 0.5 & 321 & 1.4 \\
\hline GCS 3-2 & 3 & 121 & 4.79 & 11.0 & 5.0 & 10.9 & 0.13 & 0.4 & 467 & 3.0 \\
\hline $\mathrm{J} 1743$ & 0.3 & 74 & 0.006 & 13.0 & 8.8 & 5.0 & 0.05 & 0.7 & 212 & 0.6 \\
\hline J1747 & 1 & 45 & 0.004 & 5.8 & 3.1 & 4.3 & 0.05 & 0.5 & 391 & 1.7 \\
\hline NHS 21 & 1 & 53 & 0.2 & 7.9 & 4.4 & 5.5 & 0.06 & 0.5 & 354 & 1.5 \\
\hline NHS 22 & 1 & 78 & 0.08 & 14.8 & 8.6 & 9.7 & 0.11 & 0.5 & 323 & 1.4 \\
\hline NHS 25 & 3 & 108 & 0.08 & 9.6 & 4.4 & 9.5 & 0.11 & 0.3 & 474 & 3.0 \\
\hline NHS 42 & 0.7 & 76 & 0.1 & 15.8 & 9.9 & 8.6 & 0.09 & 0.6 & 278 & 1.0 \\
\hline Mean & 1 & 66 & 0.5 & 11.2 & 6.4 & 7.5 & 0.08 & 0.5 & 337 & 1.4 \\
\hline \multicolumn{11}{|c|}{$n_{\mathrm{H}}=20 \mathrm{~cm}^{-3}$} \\
\hline Source & $\zeta$ & $L$ & $\chi^{2}$ & $N(1,1)$ & $N(1,0)$ & $N(3,3)$ & $N(2,2)$ & $f$ & $\langle T\rangle$ & $\left\langle x_{\mathrm{e}}\right\rangle$ \\
\hline GC IRS 21 & 3 & 51 & 0.37 & 22.0 & 12.6 & 17.0 & 0.35 & 0.5 & 319 & 1.3 \\
\hline GC IRS 3 & 6 & 61 & 0.46 & 10.1 & 4.5 & 11.1 & 0.25 & 0.4 & 472 & 3.0 \\
\hline GC IRS 1W & 3 & 41 & 0.19 & 17.0 & 9.8 & 12.9 & 0.26 & 0.5 & 315 & 1.3 \\
\hline GCS 3-2 & 3 & 34 & 4.90 & 12.8 & 7.3 & 9.7 & 0.20 & 0.5 & 320 & 1.4 \\
\hline $\mathrm{J} 1743$ & 0.6 & 33 & 0.01 & 12.5 & 8.6 & 4.9 & 0.10 & 0.7 & 187 & 0.6 \\
\hline J1747 & 3 & 22 & 0.21 & 6.6 & 3.6 & 5.2 & 0.11 & 0.5 & 337 & 1.6 \\
\hline NHS 21 & 2 & 25 & 0.17 & 8.4 & 4.7 & 6.3 & 0.13 & 0.5 & 320 & 1.4 \\
\hline NHS 22 & 2 & 37 & 0.15 & 15.8 & 9.3 & 11.1 & 0.23 & 0.6 & 299 & 1.2 \\
\hline NHS 25 & 3 & 29 & 0.07 & 10.4 & 5.9 & 7.9 & 0.17 & 0.5 & 321 & 1.4 \\
\hline NHS 42 & 1 & 35 & 0.14 & 16.2 & 10.5 & 8.7 & 0.18 & 0.6 & 238 & 0.8 \\
\hline Mean & 3 & 33 & 0.08 & 12.2 & 6.9 & 9.3 & 0.19 & 0.5 & 321 & 1.4 \\
\hline \multicolumn{11}{|c|}{$n_{\mathrm{H}}=50 \mathrm{~cm}^{-3}$} \\
\hline Source & $\zeta$ & $L$ & $\chi^{2}$ & $N(1,1)$ & $N(1,0)$ & $N(3,3)$ & $N(2,2)$ & $f$ & $\langle T\rangle$ & $\left\langle x_{\mathrm{e}}\right\rangle$ \\
\hline GC IRS 21 & 12 & 24 & 0.1 & 16.0 & 8.0 & 17.7 & 0.84 & 0.4 & 432 & 2.1 \\
\hline GC IRS 3 & 12 & 12 & 0.5 & 7.0 & 3.5 & 7.7 & 0.37 & 0.4 & 424 & 2.2 \\
\hline GC IRS 1W & 6 & 14 & 0.2 & 16.5 & 9.9 & 13.1 & 0.59 & 0.6 & 302 & 1.1 \\
\hline GCS 3-2 & 12 & 15 & 4.4 & 9.4 & 4.7 & 10.4 & 0.50 & 0.4 & 426 & 2.1 \\
\hline J1743 & 3 & 10 & 0.2 & 12.1 & 8.0 & 6.6 & 0.31 & 0.7 & 220 & 0.7 \\
\hline J1747 & 5 & 7 & 0.1 & 6.4 & 3.8 & 4.9 & 0.23 & 0.6 & 295 & 1.2 \\
\hline NHS 21 & 5 & 8 & 0.2 & 7.9 & 4.7 & 6.1 & 0.28 & 0.6 & 295 & 1.2 \\
\hline NHS 22 & 5 & 12 & 0.2 & 14.9 & 9.2 & 10.7 & 0.49 & 0.6 & 278 & 1.0 \\
\hline NHS 25 & 6 & 9 & 0.1 & 9.9 & 5.8 & 7.8 & 0.36 & 0.5 & 303 & 1.2 \\
\hline NHS 42 & 3 & 12 & 0.1 & 15.6 & 1.0 & 8.8 & 0.41 & 0.7 & 227 & 0.7 \\
\hline Mean & 6 & 11 & 0.1 & 12.1 & 7.2 & 9.5 & 0.44 & 0.6 & 301 & 1.1 \\
\hline \multicolumn{11}{|c|}{$n_{\mathrm{H}}=100 \mathrm{~cm}^{-3}$} \\
\hline Source & $\zeta$ & $L$ & $\chi^{2}$ & $N(1,1)$ & $N(1,0)$ & $N(3,3)$ & $N(2,2)$ & $f$ & $\langle T\rangle$ & $\left\langle x_{\mathrm{e}}\right\rangle$ \\
\hline GC IRS 21 & 11 & 7 & 0.5 & 18.6 & 11.4 & 15.9 & 1.3 & 0.6 & 310 & 1.0 \\
\hline GC IRS 3 & 30 & 8 & 1.1 & 7.7 & 3.7 & 10.2 & 0.88 & 0.4 & 505 & 2.6 \\
\hline GC IRS 1W & 10 & 6 & 0.4 & 14.8 & 9.3 & 12.0 & 0.97 & 0.6 & 294 & 1.0 \\
\hline GCS 3-2 & 11 & 4 & 7.0 & 11.0 & 6.8 & 9.0 & 0.74 & 0.6 & 297 & 1.0 \\
\hline $\mathrm{J} 1743$ & 3 & 5 & 0.04 & 12.9 & 9.7 & 4.9 & 0.44 & 0.7 & 160 & 0.4 \\
\hline J1747 & 9 & 3 & 0.2 & 6.1 & 3.8 & 4.7 & 0.40 & 0.6 & 277 & 1.0 \\
\hline NHS 21 & 10 & 3 & 0.3 & 7.4 & 4.6 & 5.9 & 0.50 & 0.6 & 286 & 1.0 \\
\hline NHS 22 & 8 & 5 & 0.1 & 14.1 & 9.2 & 9.8 & 0.80 & 0.6 & 256 & 0.8 \\
\hline NHS 25 & 11 & 4 & 0.1 & 8.9 & 5.5 & 7.3 & 0.60 & 0.6 & 293 & 1.0 \\
\hline NHS 42 & 6 & 5 & 0.2 & 15.1 & 10.2 & 9.3 & 0.77 & 0.7 & 233 & 0.7 \\
\hline Mean & 11 & 4 & 0.07 & 10.5 & 6.4 & 8.9 & 0.73 & 0.6 & 307 & 1.1 \\
\hline
\end{tabular}

Notes. Cosmic-ray ionization rates, $\zeta$, are in $10^{-14} \mathrm{~s}^{-1}$ and sizes, $L$, in parsecs. Computed $\mathrm{H}_{3}^{+}$column densities, $N(J, K)$, are in $10^{14} \mathrm{~cm}^{-2}$. The last three columns present computed molecular fractions, mean gas temperatures in Kelvin, and mean electronic fractions in $10^{-3}$.

limit to $N\left(\mathrm{H}_{2}\right)$. With an elemental abundance $\mathrm{F} / \mathrm{H}$ of $1.8 \times 10^{-8}$, the value in the solar neighborhood, and a molecular fraction of 1 , Godard et al. (2012) find $N(\mathrm{HF}) / N\left(\mathrm{H}_{2}\right)=3.6 \times 10^{-8}$ (also reported in Neufeld et al. (2005)). This ratio is only valid for media where $Z=1$ and where $n\left(\mathrm{e}^{-}\right)=n\left(\mathrm{C}^{+}\right)$. To estimate a better ratio in the CMZ, we use Eq. (C.3) of Godard et al. (2012) without the hypothesis that the density of electrons is given by $\mathrm{C}^{+}$, with $Z=3$, and with the gas temperature, electron density, and molecular fraction derived from our best models for J1747
(Table 5). We find $N(\mathrm{HF}) / N\left(\mathrm{H}_{2}\right)=1.1 \times 10^{-7}$. Derived $N\left(\mathrm{H}_{2}\right)$ are presented in Table 6.

The chemistry of HF is included in the Meudon PDR code. Using our grid of models, we tried a $\chi^{2}$ minimization built with the column densities of $\mathrm{H}_{3}^{+}$in $(1,1),(1,0)$, and $(3,3)$ toward J1747 and N(HF) toward Sgr B2(N). None of our models reproduce these four constraints at the same time. When $N\left(\mathrm{H}_{3}^{+}, J, K\right)$ are reproduced, $N(\mathrm{HF})$ is underpredicted by a factor 4. A possible explanation could be that a fraction of $\mathrm{HF}$ 
Table 6. Observed $\mathrm{HF}$ and estimated $\mathrm{H}_{2}$ column densities toward Sgr B2(N).

\begin{tabular}{rrll}
\hline \hline $\begin{array}{r}v_{\min } \\
\left(\mathrm{km} \mathrm{s}^{-1}\right)\end{array}$ & $\begin{array}{r}v_{\max } \\
\left(\mathrm{km} \mathrm{s}^{-1}\right)\end{array}$ & $\begin{array}{l}N(\mathrm{HF}) \\
\left(\times 10^{13} \mathrm{~cm}^{-2}\right)\end{array}$ & $\begin{array}{l}N\left(\mathrm{H}_{2}\right) \\
\left(\times 10^{20} \mathrm{~cm}^{-2}\right)\end{array}$ \\
\hline-150 & -114 & $0.49 \pm 0.05$ & $0.4 \pm 0.04$ \\
-114 & -100 & $2.14 \pm 0.65$ & $1.9 \pm 0.6$ \\
-100 & -88 & $2.15 \pm 0.61$ & $1.9 \pm 0.5$ \\
-88 & -60 & $5.86 \pm 0.64$ & $5.3 \pm 0.6$ \\
\hline-150 & -60 & $10.4 \pm 1.7$ & $9.4 \pm 1.5$ \\
\hline
\end{tabular}

observed toward Sgr B2(N) be associated with denser gas than the one probed by $\mathrm{H}_{3}^{+}$(but not too dense either because of the lack of $\mathrm{CO}$ absorption in the -150 to $-60 \mathrm{~km} \mathrm{~s}^{-1}$ velocity range). On the other hand, the elemental abundance of $\mathrm{F}$ is not well understood and is a widely discussed topic. We scaled it by $Z$ but it is possible that $\mathrm{F} / \mathrm{H}$ does not scale as the other elements (see Abia et al. (2015) and references therein).

The observations of $\mathrm{OH}^{+}, \mathrm{H}_{2} \mathrm{O}^{+}$, and $\mathrm{H}_{3} \mathrm{O}^{+}$are particularly interesting since, like $\mathrm{H}_{3}^{+}$, the abundances of these molecules depend on the cosmic-ray ionization rate. Schilke et al. (2010, 2013) report the detection of the $\mathrm{H}_{2} \mathrm{O}^{+}$ion toward $\mathrm{Sgr} \mathrm{B} 2$ with Herschel, whereas Lis et al. $(2012,2014)$ report $\mathrm{H}_{3} \mathrm{O}^{+}$detection. Indriolo et al. (2015) derive their column densities in the different velocity components. With a simple analytic relationship between $N\left(\mathrm{OH}^{+}\right)$and $N\left(\mathrm{H}_{2} \mathrm{O}^{+}\right)$, they deduce a value of $\zeta \sim 10^{-14} \mathrm{~s}^{-1}$, which is compatible with our value if the proton density is $\sim 10 \mathrm{~cm}^{-3}$ and lower than our value if the proton density is higher. The oxygen chemistry is included in the Meudon PDR code, and therefore we also derive $N\left(\mathrm{OH}^{+}\right), N\left(\mathrm{H}_{2} \mathrm{O}^{+}\right)$and $N\left(\mathrm{H}_{3} \mathrm{O}^{+}\right)$together with $N\left(\mathrm{H}_{3}^{+}\right)$. In Table 7 , we report these computed column densities for the best models matching $\mathrm{H}_{3}^{+}$observations toward $\mathrm{J} 1747$, for $n_{\mathrm{H}}=10,20$, and $50 \mathrm{~cm}^{-3}$, and compare them to the observed values toward Sgr B2(N) in the -130 , $-60 \mathrm{~km} \mathrm{~s}^{-1}$ velocity range. We find a very remarkable agreement. The $n_{\mathrm{H}}=50 \mathrm{~cm}^{-3}$ model underestimates the observations only slightly. The computed column density of $\mathrm{H}_{3} \mathrm{O}^{+}$is also compatible with the observations that are reported for the lowest $(1,0)$ ortho level. So, our values of $\zeta$ deduced with $\mathrm{H}_{3}^{+}$observations are consistent with $\mathrm{OH}^{+}, \mathrm{H}_{2} \mathrm{O}^{+}$and $\mathrm{H}_{3} \mathrm{O}^{+}$observations as well.

A significant discrepancy between our models and Indriolo et al. (2015) results concerns the molecular fraction. Indriolo et al. $(2012,2015)$ estimate the molecular fraction using $\mathrm{H}_{2} \mathrm{O}^{+}$ main formation and destruction reactions

$\mathrm{OH}^{+}+\mathrm{H}_{2} \longrightarrow \mathrm{H}_{2} \mathrm{O}^{+}+\mathrm{H} \quad k_{3}=1.1 \times 10^{-9} \mathrm{~cm}^{3} \mathrm{~s}^{-1}$
$\mathrm{H}_{2} \mathrm{O}^{+}+\mathrm{H}_{2} \longrightarrow \mathrm{H}_{3} \mathrm{O}^{+}+\mathrm{H} \quad k_{4}=6.1 \times 10^{-10} \mathrm{~cm}^{3} \mathrm{~s}^{-1}$
$\mathrm{H}_{2} \mathrm{O}^{+}+\mathrm{e}^{-} \longrightarrow$ products $\quad k_{5}=4.3 \times 10^{-7}(T / 300)^{-0.5} \mathrm{~cm}^{3} \mathrm{~s}^{-1}$,

and they obtain:

$f=\frac{2 x_{\mathrm{e}} k_{5} / k_{3}}{N\left(\mathrm{OH}^{+}\right) / N\left(\mathrm{H}_{2} \mathrm{O}^{+}\right)-k_{4} / k_{3}}$.

The use of this expression relies on guesses of the electronic fraction and gas temperature. Equation (6) is used in Indriolo et al. (2015) to derive $f$ in 105 diffuse gaseous components and leads to extremely small $f$ values, lower than 0.1 for most components. For Sgr B2(N), in the $-130,-60 \mathrm{~km} \mathrm{~s}^{-1}$ velocity component, Indriolo et al. (2015) obtain $f=0.08 \pm 0.02$ whereas we find $f \sim 0.5-0.6$ for $\mathrm{J} 1747$.
Table 7. Comparison of $N\left(\mathrm{OH}^{+}\right), N\left(\mathrm{H}_{2} \mathrm{O}^{+}\right)$and $N\left(\mathrm{H}_{3} \mathrm{O}^{+}\right)$observed toward Sgr B2(N) in the $-130,-60 \mathrm{~km} \mathrm{~s}^{-1}$ velocity component to the computed values by the Meudon PDR code for the J1747 best models.

\begin{tabular}{lllll}
\hline \hline & Observations $^{a}$ & \multicolumn{3}{c}{ Best models for J1747 $^{2}$} \\
& Sgr B2(N) & $10 \mathrm{~cm}^{-3}$ & $20 \mathrm{~cm}^{-3}$ & $50 \mathrm{~cm}^{-3}$ \\
\hline$\zeta$ & & 1.0 & 2.6 & 5.0 \\
$L$ & 45 & 22 & 7 \\
\hline$N\left(\mathrm{OH}^{+}\right)$ & $7.5 \pm 1.5$ & 7.1 & 8.2 & 4.1 \\
$N\left(\mathrm{H}_{2} \mathrm{O}^{+}\right)$ & $2.2 \pm 0.4$ & 2.3 & 2.4 & 1.6 \\
$N\left(\mathrm{H}_{3} \mathrm{O}^{+}\right)$ & $4.0 \pm 0.8$ & 5.1 & 5.4 & 4.9 \\
\hline
\end{tabular}

Notes. Column densities for $\mathrm{OH}^{+}$and $\mathrm{H}_{2} \mathrm{O}^{+}$are in $10^{14} \mathrm{~cm}^{-2}$ and in $10^{13} \mathrm{~cm}^{-2}$ for $\mathrm{H}_{3} \mathrm{O}^{+}$. Cosmic-ray ionization rate is in $10^{-14} \mathrm{~s}^{-1}$ and size is in pc.

References. ${ }^{(a)}$ Indriolo et al. (2015). The reported value of $N\left(\mathrm{H}_{3} \mathrm{O}^{+}\right)$is for the $(1,0)$ ortho level.

The molecular fraction may also be directly derived from observations of $\mathrm{H}$ at $21 \mathrm{~cm}$ and estimate of $N\left(\mathrm{H}_{2}\right)$ (Table 6). The column density of $\mathrm{H}$ is reported as $(1.03 \pm 0.3) \times 10^{21} \mathrm{~cm}^{-2}$ in the $-130,-60 \mathrm{~km} \mathrm{~s}^{-1} \mathrm{CMZ}$ velocity component of the Sgr B2(N) line of sight by Indriolo et al. (2015), whereas Godard et al. (2012) report $N\left(\mathrm{H}_{2}\right)=(2.0 \pm 0.5) \times 10^{21} \mathrm{~cm}^{-2}$. We previously re-estimate this value to be $(9.4 \pm 1.5) \times 10^{20} \mathrm{~cm}^{-2}$. The resulting molecular fraction is then 0.6 (0.8 with the Godard et al. (2012) estimate). These molecular fractions are then in very good agreement with those computed by the Meudon PDR code.

The origin of the discrepancy arises from the estimate of $T$ and $x_{\mathrm{e}}$. Indriolo et al. (2015) assume a gas temperature of $70 \mathrm{~K}$ and an electronic fraction of $1.5 \times 10^{-4}$, whereas the computed values in the PDR models that match J1747 and Sgr B2(N) observations are $\left\langle x_{\mathrm{e}}\right\rangle=1-2 \times 10^{-3}$ and $\langle T\rangle=295$ to $391 \mathrm{~K}$ for $n_{\mathrm{H}}$ from 50 to $10 \mathrm{~cm}^{-3}$. Using these values in Eq. (6) leads to $f \simeq 0.3-0.6$. These conflicting conclusions illustrate our discussion in Sect. 2 in which we highlight the risks of using speculated values in simple chemical analytic expressions, like Eqs. (4) or (6), especially in exotic conditions such as the Galactic center.

\subsection{Heating and cooling mechanisms}

Since the discovery of excited metastable $\mathrm{H}_{3}^{+}$in the $\mathrm{CMZ}$, several authors tried to determine the energy source that heats the gas at a few hundred Kelvin (Yusef-Zadeh et al. 2013). Several hypothesis have been investigated: photoelectric effect, cosmic rays, X-rays, turbulence, or shocks.

Figure 16 shows gas temperature, heating, and cooling rates versus the position (in $A_{\mathrm{V}}$ ) for two models with $n_{\mathrm{H}}=100 \mathrm{~cm}^{-3}$ illuminated on both sides by a radiation field that is ten times higher than the ISRF, with a metallicity $Z=3$ and with, for the first model, $\zeta=1 \times 10^{-16}$ and for the second model, $\zeta=7 \times 10^{-14} \mathrm{~s}^{-1}$. We see that for $\zeta=10^{-16} \mathrm{~s}^{-1}$, the only efficient heating mechanism is the photoelectric effect on grains. The temperature does not exceed $140 \mathrm{~K}$ and is not sufficient to excite $\mathrm{H}_{3}^{+}$as observed in CMZ conditions. On the contrary, for $\zeta=7 \times 10^{-14} \mathrm{~s}^{-1}$, cosmic-ray ionizations and exothermic chemical reactions $\left(\Delta E=4.7 \mathrm{eV}\right.$ for the $\mathrm{H}_{3}^{+}+\mathrm{e}^{-}$into the three $\mathrm{H}$ recombination channel) are efficient heating mechanisms through the whole cloud and keep the gas temperature at $T>240 \mathrm{~K}$. We find that our $4 \mathrm{eV}$ injection per cosmic-ray $\mathrm{H}_{2}$ ionization hypothesis is sufficient to heat the gas at temperatures around 250 $300 \mathrm{~K}$, consistent with $\mathrm{H}_{3}^{+}$excitation under $\mathrm{CMZ}$ conditions. 

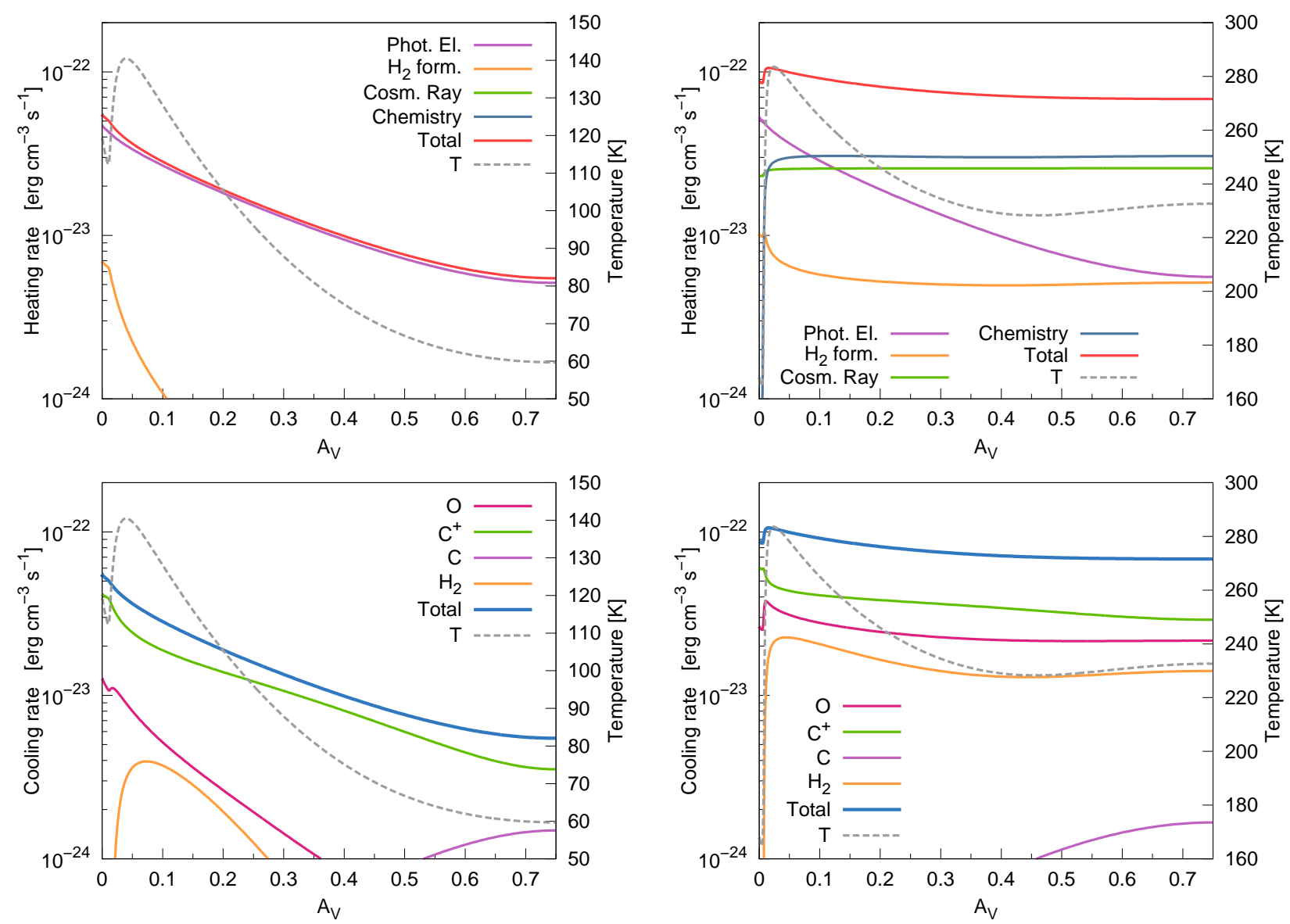

Fig. 16. Heating (top panels) and cooling (bottom panels) rates as a function of visual extinction for two models (left: $\zeta=10^{-16} \mathrm{~s}^{-1} ;$ right: $\zeta=7 \times 10^{-14} \mathrm{~s}^{-1}$ ). In both models, $n_{\mathrm{H}}=100 \mathrm{~cm}^{-3}, G_{0}=10$ and, on both sides of the clouds, $A_{\mathrm{V}}^{\max }=1.5, Z=3$. Figures present profiles from the edge of the cloud to the center. Cosmic ray heating and heating by exothermic reactions do not appear for the $\zeta=10^{-16} \mathrm{~s}^{-1}$ model because their rate is always lower than $10^{-24} \mathrm{erg} \mathrm{cm}^{-3} \mathrm{~s}^{-1}$.

The major coolants are $\mathrm{O}$ and $\mathrm{C}^{+}$in the $\zeta=10^{-16} \mathrm{~s}^{-1}$ model. For the $\zeta=7 \times 10^{-14} \mathrm{~s}^{-1}$ model, cooling by $\mathrm{H}_{2}$ starts to be important because of the high temperature. We find $\mathrm{CO}$ is not an important coolant in such diffuse gas because of a too low abundance.

\subsection{Limits and hypothesis of our models}

Our models have several limits. First, we use 1D isochoric PDR models. So, even though we account for detailed physical and chemical processes, the geometry is simplistic compared to reality. In particular, we assume only one $\mathrm{H}_{3}^{+}$component on each line of sight whereas HF absorption spectra show several components. For the $\mathrm{J} 1747$ line of sight, we tried a $\chi^{2}$ minimization on subcomponents assuming the same repartition of $\mathrm{H}_{3}^{+}$as HF. Cosmic-ray ionization rates deduced this way are on the same order of magnitude as that obtained considering only a single component.

An important factor of our models is the efficient $\mathrm{H}_{2}$ formation rate on PAHs. Our PDR models reproduce $N\left(\mathrm{H}_{3}^{+}\right)$and its excitation at high $\zeta$ because we assume $\mathrm{H}$ atoms can efficiently form $\mathrm{H}_{2}$ on PAHs via the Eley-Rideal mechanism. The formation rate of $\mathrm{H}_{2}$ on PAHs is not well known, but some recent experiments suggest the process is efficient on the edge sites of PAHs (Goumans \& Bromley 2011; Boschman et al. 2012; Mennella et al. 2012). Moreover, ISO and Spitzer observations of $\mathrm{H}_{2}$ emission lines in PDRs also suggest that $\mathrm{H}_{2}$ must be formed efficiently on PAHs (Habart et al. 2004, 2011). If the $\mathrm{H}_{2}$ formation rate was lower than that computed in our models, the deduced cosmic-ray flux would be lower (see Fig. 7) and not efficient enough to heat the gas and explain $\mathrm{H}_{3}^{+}$excitation. Then, other heating sources would have to be found. We tested several other possibilities, and none of them are satisfying. Increasing the UV flux to heat the gas by photoelectric effect on grains is not a good hypothesis as the photodissociation rate of $\mathrm{H}_{2}$ becomes too high, such that it becomes difficult to reproduce the observed column densities. We also investigated the role of X-rays heating with a new version of the Meudon PDR code (Godard et al., in prep.). Several X-rays sources can be found in the Galactic center. X-rays emitted by the ionized gas in the central cavity have a flux that is too low to be responsible for the gas temperature in the CMZ (the most distant excited $\mathrm{H}_{3}^{+}$detected by Goto et al. 2011 is at more than $100 \mathrm{pc}$ of Sgr $\mathrm{A}^{*}$ ). Another candidate could be heating by turbulent energy dissipation. Thanks to their vortex model, the TDR code, Godard et al. (2014) suggested that intermittent turbulent energy dissipation may explain the presence of $\mathrm{SH}^{+}$and $\mathrm{CH}^{+}$observed by Herschel in the CMZ. Nevertheless, this process only heats a small fraction of the gas and may not be efficient enough to explain the global heating of the CMZ. Introducing this scenario for $\mathrm{H}_{3}^{+}$would require a numerical model consistently taking both PDR physics and chemistry into account as well as turbulent energy dissipation effects on chemistry and $\mathrm{H}_{3}^{+}$excitation. E. Bron (Bron 2014) developed a promising method to combine both approaches, based on the 
modeling of statistical properties of turbulence ${ }^{7}$. We will test this method in a future study.

\section{Conclusions}

Our PDR models suggest a cosmic-ray ionization rate of $\mathrm{H}_{2}$ in the CMZ of $\sim(1-11) \times 10^{-14} \mathrm{~s}^{-1}$. The origin of this uncertainty range is due to the difficulty in constraining density from observations. This high value allows us to reproduce the observed column densities of $\mathrm{H}_{3}^{+}$and its unusual excitation in the metastable state $(3,3)$ observed toward nine lines of sight in the CMZ spanning $\sim 100 \mathrm{pc}$ apart from Sgr A*. The deduced cosmic-ray ionization rate is also compatible with the one deduced from synchrotron emission and $\mathrm{Fe} \mathrm{K} \alpha$ line emission (Yusef-Zadeh et al. 2013). As a bonus, we also reproduce $N\left(\mathrm{OH}^{+}\right), N\left(\mathrm{H}_{2} \mathrm{O}^{+}\right)$and $N\left(\mathrm{H}_{3} \mathrm{O}^{+}\right)$observed in the CMZ by Herschel toward Sgr B2(N), a line of sight close to one line of sight where $\mathrm{H}_{3}^{+}$is detected. We confirm the suggestion by Oka \& Epp (2004) that the gas probed by $\mathrm{H}_{3}^{+}$observations in the $\mathrm{CMZ}$ is diffuse and warm. Indeed, PDR models matching observations have a proton density $\lesssim 100 \mathrm{~cm}^{-3}$ and $T \sim 212-505 \mathrm{~K}$. The main source of heating of the diffuse gas in the CMZ is the suprathermal electrons ejected from the gas by cosmic-ray ionizations and exothermic dissociative recombination reactions. If the analysis is only based on the J1747 line of sight, for which more constraints can be used, then models tend to favor gas densities that are lower than $50 \mathrm{~cm}^{-3}$. In that case, sizes of the diffuse components probed by $\mathrm{H}_{3}^{+}$are large (several ten parsecs to about 100 parsecs). This means that the diffuse gas probed by $\mathrm{H}_{3}^{+}$spans the whole CMZ. This is in agreement with $\mathrm{H}_{3}^{+}$observations that present the same behaviors in different directions in the CMZ.

We also show the importance of treating the detail of physical processes in astrochemical problems. We demonstrate how the simple analytic relationship between $N\left(\mathrm{H}_{3}^{+}\right)$and $\zeta$ (Eq. (4)), commonly used to infer the cosmic-ray ionization rate in interstellar gas, may fail in an exotic medium. Indeed, for low and moderate cosmic-ray ionization rates the column density of $\mathrm{H}_{3}^{+}$increases linearly with $\zeta$ but decreases at high $\zeta$, in contradiction with the analytic expression. This is due to the ionization of $\mathrm{H}$ and $\mathrm{H}_{2}$ by cosmic rays, which reduces the molecular fraction of the gas and increases the electronic fraction of the gas leading to fast $\mathrm{H}_{3}^{+}$recombination.

A key parameter of our results concerns the $\mathrm{H}_{2}$ formation rate. We reached the conclusion that the $\mathrm{H}_{2}$ formation rate in the diffuse gas of the CMZ must be enhanced when compared to local diffuse clouds. Indeed, in the CMZ, as in PDRs, the warm temperature of the gas opens the possibility of efficiently forming $\mathrm{H}_{2}$ on grains and $\mathrm{PAH}$ via chemisorption of $\mathrm{H}$ atoms followed by Eley-Rideal mechanism. Therefore, on the one hand, a high cosmic-ray ionization rate reduces the number of available $\mathrm{H}$ atoms to form $\mathrm{H}_{2}$ since they are ionized in $\mathrm{H}^{+}$. On the other hand, heating the gas to several hundred Kelvin, cosmic rays open the efficient $\mathrm{H}_{2}$ formation route via chemisorption sites. This allows us to reach high column densities of $\mathrm{H}_{3}^{+}$at large cosmic-ray ionization rates.

Acknowledgements. We thank Octavio Roncero who made the $\mathrm{H}_{3}^{+}-\mathrm{H}_{2}$ collision rates available to us. We thank Takeshi Oka for useful discussions about $\mathrm{H}_{3}^{+}$and $\mathrm{CO}$ in the central molecular zone. Exchanges with Farhad Yusef-Zadeh and Vincent Tatischeff are acknowledged for their assistance in deriving the cosmicray ionization rate from synchrotron emission. We also thank Mickaël Coriat for his help concerning high energy astrophysics and radio counterpart as well

\footnotetext{
Available at https: //tel . archives-ouvertes . fr/ tel-01111148
}

as Thibaut Paumard for useful discussions about the Galactic center. This work was supported in part by grant SYMPATICO (ANR-11-BS56-0023) from the French Agence Nationale de la Recherche. It was also supported by the French CNRS national program PCMI. Maxime Ruaud acknowledges funding by the European Research Council (Starting Grant 3DICE, grant agreement 336474).

\section{References}

Abia, C., Cunha, K., Cristallo, S., \& de Laverny, P. 2015, A\&A, 581, A88

Aguado, A., Barragán, P., Prosmiti, R., et al. 2010, J. Chem. Phys., 133, 024306 Bialy, S., \& Sternberg, A. 2015, MNRAS, 450, 4424

Boschman, L., Reitsma, G., Cazaux, S., et al. 2012, ApJ, 761, L33

Brittain, S. D., Simon, T., Kulesa, C., \& Rettig, T. W. 2004, ApJ, 606, 911

Bron, E. 2014, Ph.D. Thesis, Université Paris Diderot, France

Bron, E., Le Bourlot, J., \& Le Petit, F. 2014, A\&A, 569, A100

Compiègne, M., Verstraete, L., Jones, A., et al. 2011, A\&A, 525, A103

Daflon, S., \& Cunha, K. 2004, ApJ, 617, 1115

Draine, B. T., \& Li, A. 2007, ApJ, 657, 810

Faure, A., \& Tennyson, J. 2003, MNRAS, 340, 468

Faure, A., Wiesenfeld, L., Valiron, P., et al. 2006, Roy. Soc. London Phil. Trans. Ser. A, 364, 3113

Federman, S. R., Weber, J., \& Lambert, D. L. 1996, ApJ, 463, 181

Felenbok, P., \& Roueff, E. 1996, ApJ, 465, L57

Fitzpatrick, E. L., \& Massa, D. 1986, ApJ, 307, 286

Flower, D. R., \& Pineau des Forets, G. 1998, MNRAS, 297, 1182

Geballe, T. R. 2012, Roy. Soc. London Phil. Trans. Ser. A, 370, 5151

Geballe, T. R., \& Oka, T. 1996, Nature, 384, 334

Geballe, T. R., \& Oka, T. 2010, ApJ, 709, L70

Geballe, T. R., McCall, B. J., Hinkle, K. H., \& Oka, T. 1999, ApJ, 510, 251

Geballe, T. R., Goto, M., Usuda, T., Oka, T., \& McCall, B. J. 2006, ApJ, 644, 907

Gerin, M., de Luca, M., Black, J., et al. 2010a, A\&A, 518, L110

Gerin, M., de Luca, M., Goicoechea, J. R., et al. 2010b, A\&A, 521, L16

Gibb, E. L., Brittain, S. D., Rettig, T. W., et al. 2010, ApJ, 715, 757

Godard, B., Falgarone, E., Gerin, M., et al. 2012, A\&A, 540, A87

Godard, B., Falgarone, E., \& Pineau des Forêts, G. 2014, A\&A, 570, A27

Gómez-Carrasco, S., González-Sánchez, L., Aguado, A., et al. 2012, J. Chem. Phys., 137, 094303

Gonzalez Garcia, M., Le Bourlot, J., Le Petit, F., \& Roueff, E. 2008, A\&A, 485, 127

Goto, M., McCall, B. J., Geballe, T. R., et al. 2002, PASJ, 54, 951

Goto, M., McCall, B., Geballe, T., Usuda, T., \& Oka, T. 2005, in High Resolution Infrared Spectroscopy in Astronomy, eds. H. U. Käufl, R. Siebenmorgen, \& A. Moorwood (ESO Astrophysics Symposia), 244

Goto, M., Usuda, T., Nagata, T., et al. 2008, ApJ, 688, 306

Goto, M., Usuda, T., Geballe, T. R., et al. 2011, PASJ, 63, L13

Goto, M., Indriolo, N., Geballe, T. R., \& Usuda, T. 2013, J. Phys. Chem. A, 117, 9919

Goto, M., Geballe, T. R., Indriolo, N., et al. 2014, ApJ, 786, 96

Goumans, T. P. M., \& Bromley, S. T. 2011, MNRAS, 414, 1285

Grenier, I. A., Black, J. H., \& Strong, A. W. 2015, ARA\&A, 53, 199

Gry, C., Boulanger, F., Nehmé, C., et al. 2002, A\&A, 391, 675

Habart, E., Boulanger, F., Verstraete, L., Walmsley, C. M., \& Pineau des Forêts, G. 2004, A\&A, 414, 531

Habart, E., Abergel, A., Boulanger, F., et al. 2011, A\&A, 527, A122

Hartquist, T. W., Black, J. H., \& Dalgarno, A. 1978, MNRAS, 185, 643

Hugo, E., Asvany, O., \& Schlemmer, S. 2009, J. Chem. Phys., 130, 164302

Indriolo, N., \& McCall, B. J. 2012, ApJ, 745, 91

Indriolo, N., Geballe, T. R., Oka, T., \& McCall, B. J. 2007, ApJ, 671, 1736

Indriolo, N., Blake, G. A., Goto, M., et al. 2010, ApJ, 724, 1357

Indriolo, N., Neufeld, D. A., Gerin, M., et al. 2012, ApJ, 758, 83

Indriolo, N., Neufeld, D. A., Gerin, M., et al. 2015, ApJ, 800, 40

Jura, M. 1974, ApJ, 191, 375

Kokoouline, V., Faure, A., Tennyson, J., \& Greene, C. H. 2010, MNRAS, 405, 1195

Le Bourlot, J., Le Petit, F., Pinto, C., Roueff, E., \& Roy, F. 2012, A\&A, 541, A76 Le Petit, F., Roueff, E., \& Herbst, E. 2004, A\&A, 417, 993

Le Petit, F., Nehmé, C., Le Bourlot, J., \& Roueff, E. 2006, ApJS, 164, 506

Le Petit, F., Barzel, B., Biham, O., Roueff, E., \& Le Bourlot, J. 2009, A\&A, 505, 1153

Lindsay, C. M., \& McCall, B. J. 2001, J. Mol. Spectr., 210, 60

Lis, D. C., Schilke, P., Bergin, E. A., \& Emprechtinger, M. 2012, Roy. Soc. London Phil. Trans. Ser. A, 370, 5162

Lis, D. C., Schilke, P., Bergin, E. A., et al. 2014, ApJ, 785, 135

Liszt, H. 2003, A\&A, 398, 621

Liszt, H. S. 2006, Roy. Soc. London Phil. Trans. Ser. A, 364, 3049

Mathis, J. S., Mezger, P. G., \& Panagia, N. 1983, A\&A, 128, 212 
Mathis, J. S., Rumpl, W., \& Nordsieck, K. H. 1977, ApJ, 217, 425

McCall, B. J., Geballe, T. R., Hinkle, K. H., \& Oka, T. 1998, Science, 279, 1910

McCall, B. J., Geballe, T. R., Hinkle, K. H., \& Oka, T. 1999, ApJ, 522, 338

McCall, B. J., Hinkle, K. H., Geballe, T. R., et al. 2002, ApJ, 567, 391

McCall, B. J., Huneycutt, A. J., Saykally, R. J., et al. 2003, Nature, 422, 500

Mennella, V., Hornekær, L., Thrower, J., \& Accolla, M. 2012, ApJ, 745, L2

Meyer, D. M., Cardelli, J. A., \& Sofia, U. J. 1997, ApJ, 490, L103

Meyer, D. M., Jura, M., \& Cardelli, J. A. 1998, ApJ, 493, 222

Moskalenko, I. V., Porter, T. A., \& Strong, A. W. 2006, ApJ, 640, L155

Neufeld, D. A., Wolfire, M. G., \& Schilke, P. 2005, ApJ, 628, 260

Neufeld, D. A., Goicoechea, J. R., Sonnentrucker, P., et al. 2010, A\&A, 521, L10

Oka, T. 2013, Chem. Rev., 113, 8738

Oka, T. 2015, in AIP Conf. Ser., 1642, 373

Oka, T., \& Epp, E. 2004, ApJ, 613, 349

Oka, T., Geballe, T. R., Goto, M., Usuda, T., \& McCall, B. J. 2005, ApJ, 632, 882

Oka, T., Onodera, Y., Nagai, M., et al. 2012, ApJS, 201, 14
Park, K., \& Light, J. C. 2007, J. Chem. Phys., 126, 044305

Porter, T. A., \& Strong, A. W. 2005, Inter. Cosmic Ray Conf., 4, 77

Rachford, B. L., Snow, T. P., Tumlinson, J., et al. 2002, ApJ, 577, 221

Rolleston, W. R. J., Smartt, S. J., Dufton, P. L., \& Ryans, R. S. I. 2000, A\&A, 363,537

Roueff, E. 1996, MNRAS, 279, L37

Rudolph, A. L., Fich, M., Bell, G. R., et al. 2006, ApJS, 162, 346

Savage, B. D., \& Sembach, K. R. 1996, ARA\&A, 34, 279

Schilke, P., Comito, C., Müller, H. S. P., et al. 2010, A\&A, 521, L11

Schilke, P., Lis, D. C., Bergin, E. A., Higgins, R., \& Comito, C. 2013, J. Phys. Chem. A, 117, 9766

Seaton, M. J. 1979, MNRAS, 187, 73

Snow, T. P., Destree, J. D., \& Jensen, A. G. 2007, ApJ, 655, 285

Sternberg, A., Le Petit, F., Roueff, E., \& Le Bourlot, J. 2014, ApJ, 790, 10

Strong, A. W., Porter, T. A., Digel, S. W., et al. 2010, ApJ, 722, L58

Wakelam, V., Herbst, E., Loison, J.-C., et al. 2012, ApJS, 199, 21

Yusef-Zadeh, F., Hewitt, J. W., Wardle, M., et al. 2013, ApJ, 762, 33 